\title{
Tremor migration patterns and the collective behavior of deep asperities mediated by creep
}

\author{
Yingdi Luo ${ }^{1}$ and Jean-Paul Ampuero ${ }^{1}$ \\ ${ }^{1}$ Seismological Laboratory, California Institute of Technology, CA 91125, USA
}

\begin{abstract}
Slow-slip events (SSE) and non-volcanic tremors have revealed a broad spectrum of earthquake behavior, involving entangled seismic and aseismic slip, and offer a unique window into fault mechanics at the bottom of seismogenic zones. A hierarchy of migration patterns of tremors has been observed in the Cascadia subduction zone, including large-scale along-strike tremor propagation and Rapid Tremor Reversals (RTR) migrating in opposite directions with much higher propagation speeds. Here we show that these tremor migration patterns can be reproduced by two end-member models of a fault with heterogeneous mechanical properties, composed of competent asperities embedded in a more frictionally stable, incompetent matrix. In the SSE-driven-tremor model, SSEs are spontaneously generated by the matrix, even in absence of seismic asperities, and drive tremor. In the tremor-driven-SSE model the matrix is stable, it slips steadily in absence of asperities, and SSEs result from the collective behavior of tremor asperities interacting via transient creep in the form of local afterslip fronts. We study these two end-member models through 2D quasi-dynamic multi-cycle simulations of faults governed by rate-and-state friction with heterogeneous frictional properties and effective normal stress, using the earthquake simulation software QDYN (Luo et al. 2017). In both models, tremor migration patterns emerge from interactions between asperities mediated by creep transients. The models successfully reproduce forward tremor propagation and RTRs, as well as various other observed tremor migration patterns, without the need to finely tune model parameters. Our modeling results suggest that, in contrast to a common view, SSE could be a result of tremor activity. Also, the hierarchical pattern of tremor migrations provides general constraints on fault zone rheology, and the location of RTRs and other tremor patterns might shed light on the finer scale spatial variability of fault properties. We also find that, despite important interactions between asperities, tremor activity rates are proportional to the underlying aseismic slip rate, supporting an approach to estimate SSE properties with high spatial-temporal resolutions via tremor activity.
\end{abstract}

\section{Introduction}

Slow-slip events (SSEs) and non-volcanic tremors (NVTs) discovered in the last decade (Rogers and Dragert 2003) have greatly expanded the spectrum of observed earthquake behavior (Beroza and Ide 2011; Gomberg 2010; Hawthorne and Rubin 2010; Ide et al. 2007a; Peng and Gomberg 2010). SSEs are aseismic slip transients with a small slip rate (few orders of magnitude larger than the plate loading rate, well below seismic slip rate), they evolve more slowly and have a much longer duration than regular earthquakes, and have been mostly observed near the deep seismic-aseismic transition (30 to $50 \mathrm{~km}$ ) below the conventional seismogenic zone depth. Tremors are emergent and continuous seismic signals, with relatively small amplitude and energy concentrated at frequencies between 1 and $10 \mathrm{~Hz}$, lower than regular earthquakes of comparable magnitudes (whose energy extends above $10 \mathrm{~Hz}$ ). Tremors are often composed of multiple Low Frequency Earthquakes (LFEs), apparently repeated failures of a same (or closely located) fault asperity (e.g. Shelly et al. 2007). Tremors are often organized in swarms that migrate. An imbricated hierarchy of tremor migration patterns has been observed in the Cascadia subduction zone (Figure 1) during each recurring episodic tremor and slow-slip event (ETS): large-scale forward tremor propagation along the fault strike direction at about 5-10 km/day, sparsely distributed swarms that propagate about 5 to 50 times faster in the opposite direction ("rapid tremor reversals" 
or RTRs) (Houston et al. 2011), and tremor swarms that propagate even faster along-dip in the vicinity of the main SSE front (Ghosh et al. 2010; Peng et al. 2015). Bletery et al. (2017) found that secondary tremor fronts slow down as they propagate. Other tremor migration patterns have also been observed, such as tremor halting and branching, acceleration and deceleration (Kao et al. 2009). Hierarchical tremor migration patterns have also been observed in other subduction zones, such as in Shikoku, Japan (Shelly et al. 2006, 2007). Lengliné et al. (2017) studied the inter-event times between LFE repeats in the Cascadia and Mexico subduction zones and found they decay inversely proportional to time after their first occurrence.
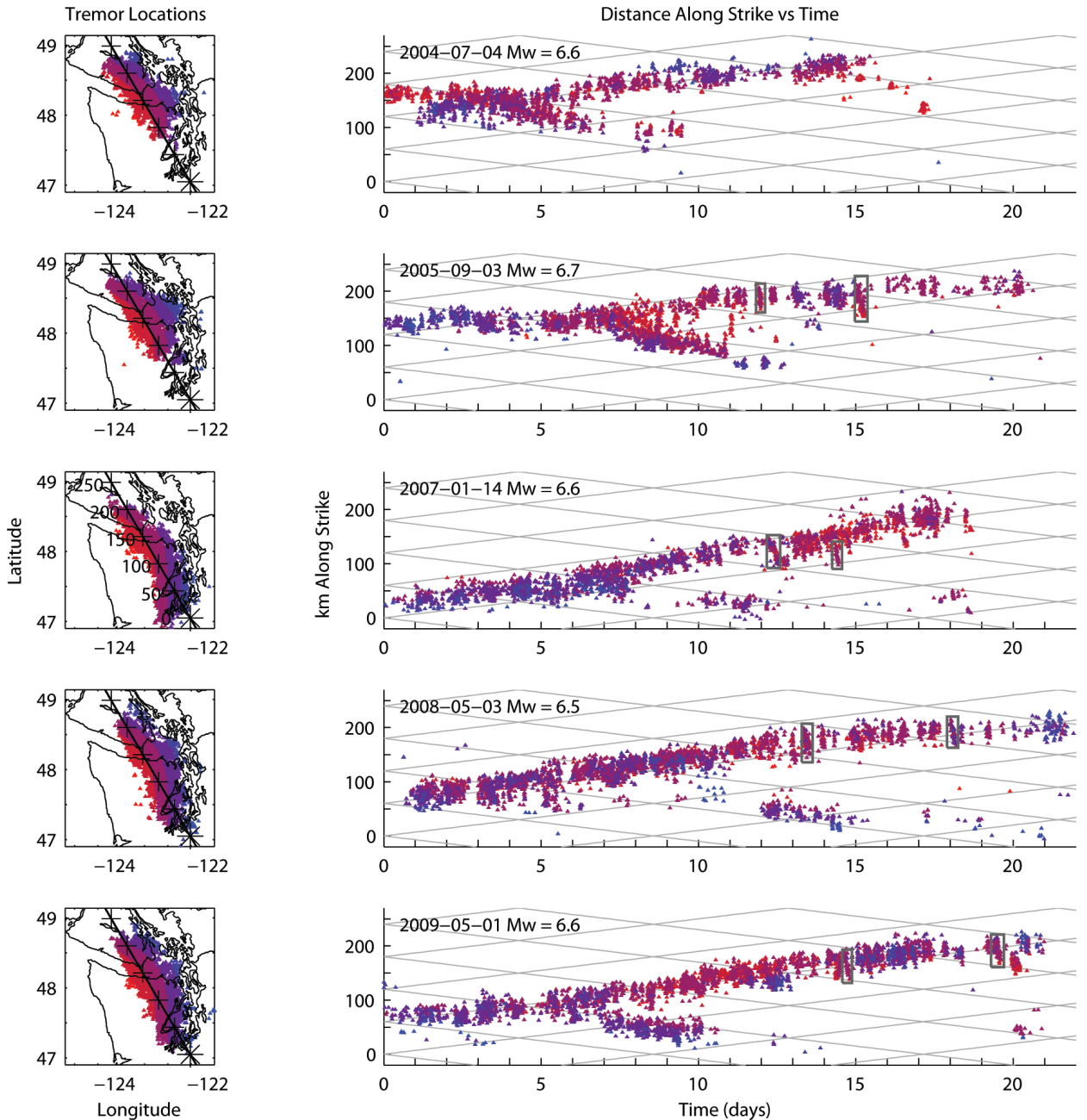

Figure 1: Observations of tremor migration patterns, courtesy of H. Houston. Right: spatial-temporal distribution of tremors during each ETS, projected along-strike, showing large-scale forward tremor migration and sparsely distributed rapid tremor reversals (selected RTRs marked with gray rectangles). Left: map view.

The study of SSE and tremor is significant for fundamental and practical reasons. It offers a unique window into the mechanics of the bottom of the seismogenic zone, and may contribute to earthquake hazard assessment. The location of tremor and SSE constrains the deep transition from seismogenic to aseismic slip; it may provide bounds on seismogenic width and on the maximum depth extent of large earthquake ruptures. Such information is critical in subduction regions: it controls the proximity of the source to dense urban areas, hence the potential impact of ground motions. Despite various modeling efforts (e.g. Ariyoshi et al. 2009, 2012; Ben-Zion 2012; Colella et al. 2011, 2013; 
Daub et al. 2011; Gershenzon et al. 2011; Hawthorne and Rubin 2013a,b; Ide et al. 2008; Rubin 2011), the nature of tremor and SSE phenomena is still incompletely understood. Most existing models reproduce subsets of the observed phenomena, some operate successfully only within a narrow range of model parameter values and, most importantly, none of those models are general enough to reproduce quantitatively the observed patterns of both SSE and tremors (see section 4.4). Thus a study of imbricated tremor and SSE processes can help understand fundamental aspects of fault mechanics, for instance by providing constraints on the rheology of the seismic-aseismic transition zones of major faults. The study of SSE and tremors might also help understanding large earthquakes. ETS in Cascadia and Shikoku are clearly segmented along strike. Several ETS cycles have been observed on each segment, including events that span multiple segments. Accounting for multi-segment ruptures is an important recent development in earthquake hazard assessment (Field et al. 2017). The study of ETS segmentation may help constrain mechanical and statistical models of multi-segment interactions. Observing and modeling ETS may also shed light on the slow slip and foreshock activity that has been observed or inferred to precede some recent large subduction earthquakes, such as the 2014 Iquique, Chile earthquake (Ampuero et al. 2014; Ruiz et al. 2014) and the 2011 Tohoku, Japan earthquake (Kato et al. 2012)). Tremor activity during the initiation of large SSEs also deserves focused studies, as it may be a slow-motion version of the nucleation process of large earthquakes.

Studying the relation between SSE and tremor may reveal new approaches to monitor SSE through tremor activity. SSEs are conventionally monitored by geodetic methods, such as GPS, which have limited spatial resolution on deep slip (tens of kilometers) and limited detection capability for very slow slip rates (microns per second). The coupling between SSEs and tremors provides observations with higher resolution. Advances in data analysis techniques, including beam-forming of multiple arrays (Ghosh et al. 2010), waveform matched filter LFE detection (Shelly et al. 2007) and detection algorithms utilizing current developments such as machine learning (Rouet-Leduc et al. 2017), are allowing the characterization of tremor activity with increasing resolution. Thus it is timely to study the mechanical relations between the spatial-temporal behavior of non-volcanic tremors and the underlying slow slip transients. We will show (in section 4.3) that in our model the tremor rate is proportional to local slip rates. Such a relation is straightforward to conceive for isolated asperities producing repeating earthquakes, and it has been commonly assumed or inferred that the seismicity rate of repeating earthquakes is proportional to the slip rate of the driving aseismic slip (e.g. Chen et al. 2007). However, in a fault containing multiple closely located asperities, such as in the tremor environment, it is uncertain to what degree the interactions between asperities complicate the relation between seismic and aseismic activity. Our models allow us to investigate the relation between tremor rate and slip rate under strong interactions, to understand how to constrain SSE slip rate through tremor observations.

Here, we will address four key open questions about tremor and SSE processes:

1. Is SSE a cause or a result of tremor? In other words, do tremors just ride the wave of a slow slip event, or do they contribute to, or even generate slow slip? To what extent are tremors and slow slip two inextricable manifestations of a single mechanical process? To address this fundamental question, we design two end-member models: in the first model tremor activities are driven by slow slip, while in the second model slow slip is driven by tremor. We aim at identifying differences that can distinguish these two models in current or future observational studies. While currently the common view is that tremors are the byproduct of SSEs, we will show that some observed characteristics of tremor migration patterns actually favor the tremor-driven-SSE model.

2. What do tremor migration patterns tell us about fault rheology? Migrating tremor swarms display intriguing hierarchical patterns. We will find that the imbricated patterns of forward tremor migration and RTR shed light on the rheology of the fault zone matrix. For instance, we will find that linear viscosity is insufficient to generate hierarchical tremor migration patterns.

3. Can a heterogeneous rate-and-state fault generate SSEs robustly, without fine tuning of model parameters? Previous studies show that a homogeneous fault governed by the classical, laboratory-motivated rate-and-state friction law (defined in section 2) can generate SSEs only within a narrow range of model parameters (Rubin 2008). The range is especially narrow with the "slip law", the state evolution law that is most consistent with laboratory experiments with large velocity steps. This fine tuning issue has motivated the study of other physical ingredients to explain the generation of SSE, including more sophisticated friction laws with transitions from velocity-weakening to velocity-strengthening at increasing slip rate (Hawthorne and Rubin 2013a) and the hardening effect of fault gouge dilatancy in fluid-saturated fault zones (Segall et al. 2010). Here we will investigate if fault heterogeneity can lead to robust SSEs under classical rate-and-state friction with the slip law. 
4. Can SSE properties be inferred from tremor activity? We will demonstrate that the SSE slip rate and tremor activity rate are closely related. Thus, observed tremor activity rates can be used to estimate, with high spatial-temporal resolution, slip rates of the underlying SSE.

The remainder of this paper is organized as follows. In section 2 Modeling, we present the basic ingredients of our heterogeneous fault models, our conceptual explanation of the origin of hierarchical tremor migration patterns and general aspects of rate-and-state friction. We then introduce the two end-member models of SSE-driven-tremor and tremor-driven-SSE. In section 3 Results, we present 2D numerical rate-and-state simulation results to demonstrate that both models produce quantitative agreement with observations of SSE and tremor migration. We then analyze the effects of model parameters, such as the strength contrast and distribution of asperities, on observable tremor properties. In section 4 Discussion, we compare the two models and identify observable characteristics of tremor that might be used to distinguish them. We then establish links between RTR propagation speed and distance, and the underlying slow slip rate. We also discuss previously proposed models in light of our modeling results, review the limitations of our models, propose directions for future work, and present preliminary results of 3D modeling. Finally, in section 5 Conclusion, we summarize our results in relation to the key questions formulated above.

\section{Modeling}

\subsection{Conceptual models}

In a view that has emerged from tremor and slow slip observations (e.g. Ito et al. 2009), tremor results from dynamic shear failure of competent asperities on an otherwise creeping fault zone. The seismic-aseismic transition region has heterogeneous frictional properties, and is composed of frictionally unstable patches ("competent asperities") embedded in a frictionally more stable fault matrix. Whereas tremors are commonly viewed as swarms of LFEs driven by an underlying, larger scale slow slip transient, here we also explore how tremor swarms can emerge from the collective response of asperities that trigger aseismic slip in their surroundings and interact via these aseismic transients (Figure 2 and 3, see also Ariyoshi et al. $(2009,2012)$ ).

The existence of deep asperities is supported by seismological and geological observations. Seismological evidence includes the observation that tremors are composed of LFEs (Ide et al. 2007b; Shelly et al. 2006, 2007) and occasionally Very Low Frequency Earthquakes with double couple focal mechanism consistent with shear failure on the megathrust. The existence of deep asperities is also supported by the deepening of seismicity following the M7.3 Landers earthquake (Rolandone et al. 2004) and by high frequency radiation from the deeper parts of the fault during the 2010 Tohoku and other subduction earthquakes (Lay et al. 2012; Meng et al. 2011). Geological observations of exhumed subduction faults show a mélange structure with competent lenses embedded in a incompetent matrix (Bebout and Barton 2002; Fagereng 2011; Fagereng and Cooper 2010; Fagereng and Sibson 2010; Meneghini et al. 2010).

In our conceptual model, seismic failure of a competent asperity induces post-seismic slip that propagates outwards from the asperity. Propagating afterslip is typically obtained in numerical simulations (Figure 4, see also Ariyoshi et al. (2012) and Kato (2007)) and is supported by observations of expanding aftershock zones (Kato 2007; Peng and Gomberg 2010). An afterslip front approaching another asperity loads it and can trigger its rupture (Lui and Lapusta 2016). The process can repeat, leading to a cascade of asperity ruptures mediated by afterslip and taking the appearance of a tremor swarm (Figure 2; see also Ando et al. (2010, 2012) and Ariyoshi et al. (2009, 2012)). If asperities are triggered at the arrival of afterslip fronts, without significant delay, then the migration speed of the swarm is controlled by the propagation speed of the afterslip front, $V_{\text {prop }}$. On the one hand, a relation between the propagation speed of a slip front $V_{\text {prop }}$ and its peak slip velocity $V_{\max }$, valid for any friction law that features weakening within a small process zone near the slip front, is (Ampuero and Rubin 2008):

$$
V_{\text {prop }} \propto \frac{G V_{\text {max }}}{\Delta \tau_{p-r}}
$$

where $G$ is the shear modulus and $\Delta \tau_{p-r}$ is the peak to residual strength drop due to frictional weakening. In rate-andstate friction laws derived from laboratory experiments at low slip rate, $\Delta \tau_{p-r}$ depends only weakly (logarithmically) on $V_{\max }$, hence a key outcome of Equation 1 is:

$$
V_{\text {prop }} \propto V_{\text {max }}
$$



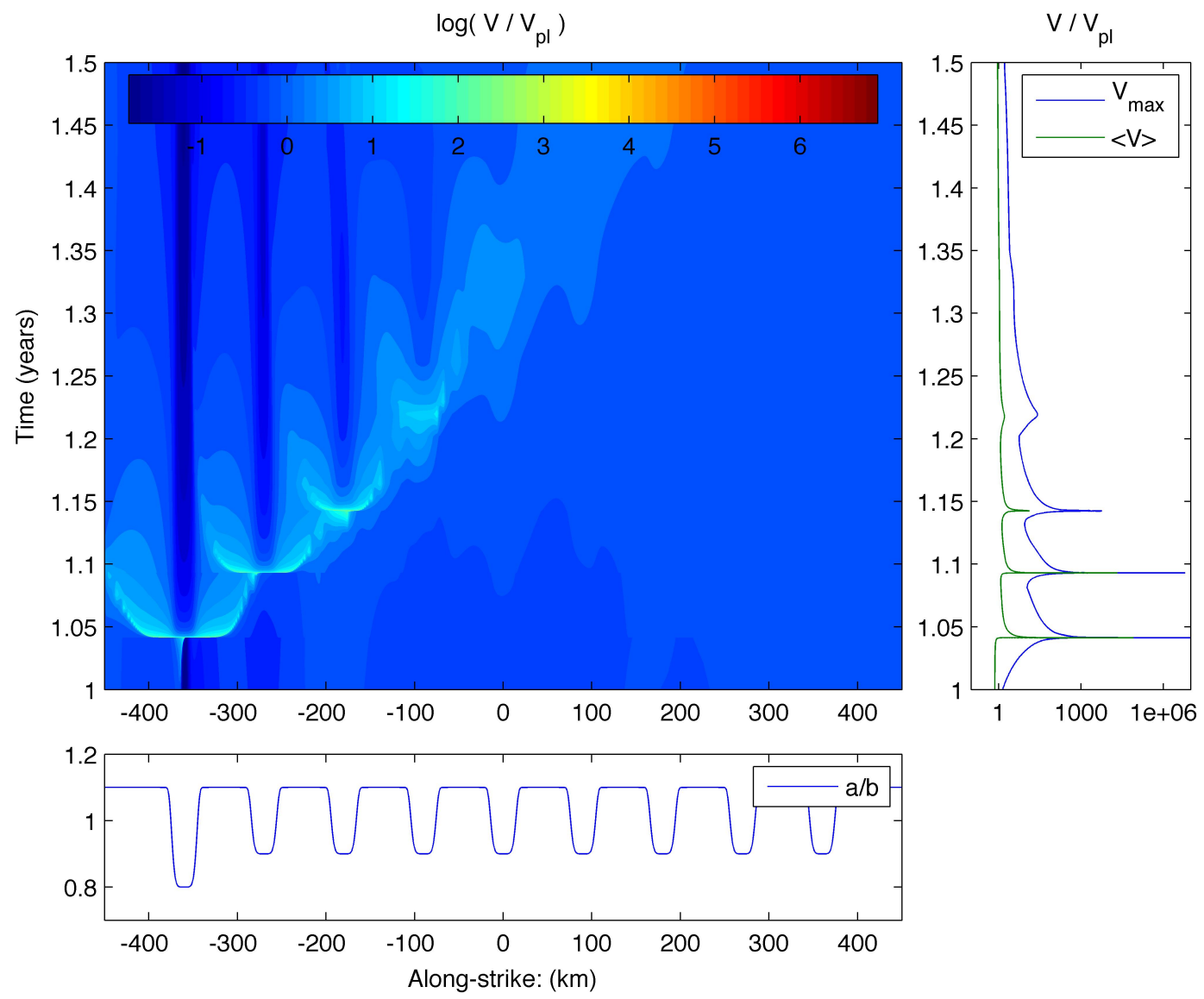

Figure 2: An example of asperity triggering cascade on a 1D fault. The main plot shows the logarith of slip rate normalized by plate velocity as a function of space and time. Right: maximum slip rate (blue) and average slip rate over the fault (green) as a function of time. Bottom: spatial distribution of $a / b$ ratio. Velocity strengthening regions have $a>b$, velocity weakening asperities have $a<b$. When the leftmost asperity fails, it produces a migrating post-seismic slip perturbation that triggers the adjacent asperity. This process repeats in a cascade.

On the other hand, if $V_{\max }$ correlates with the background slip rate $V_{b g}$ that prevails before the arrival of a slip front, then $V_{\text {prop }}$ correlates with $V_{b g}$. We will show in section 2.2 that such a correlation is satisfied by rate-and-state friction (Figure 4), but it is not satisfied by other fault zone rheologies such as the Newtonian viscosity assumed by Ando et al. $(2010,2012)$ (see more in section 4.4). For rate-and-state friction we will indeed find a nearly proportional relation, thus

$$
V_{\text {prop }} \propto V_{b g}
$$

The slip rate within an SSE pulse is the background slip rate $V_{b g}$ that prevails around the tremor asperities and, according to the relation above, controls the propagation speed $V_{\text {prop }}$. The spatial distribution of slip rate within an SSE pulse is expected, from fracture mechanics, to have high values near the front and low values at the tail. These features are illustrated in Figure 5, which shows the slip rate distribution as a function of time and distance of a sample SSE from our rate-and-state simulations (See also figure 3 and figure 4 in Hawthorne and Rubin 2013a). Thus we interpret the very fast (mostly) along-dip tremor swarms as running along the front of the SSE pulse, where the highest $V_{b g}$ values are concentrated, and RTRs as swarms running into the tail of the SSE pulse, where $V_{b g}$ is lower. The slowdown of RTRs (Bletery et al. 2017) and tremor branching are interpreted as swarms running further into the SSE pulse tail where $V_{b g}$ gradually decreases.

In this conceptual model, the qualitative connection between tremor migration speed and the underlying slow slip rate leads naturally to the whole hierarchy of tremor migration patterns (Figure 6). To make quantitative comparisons 


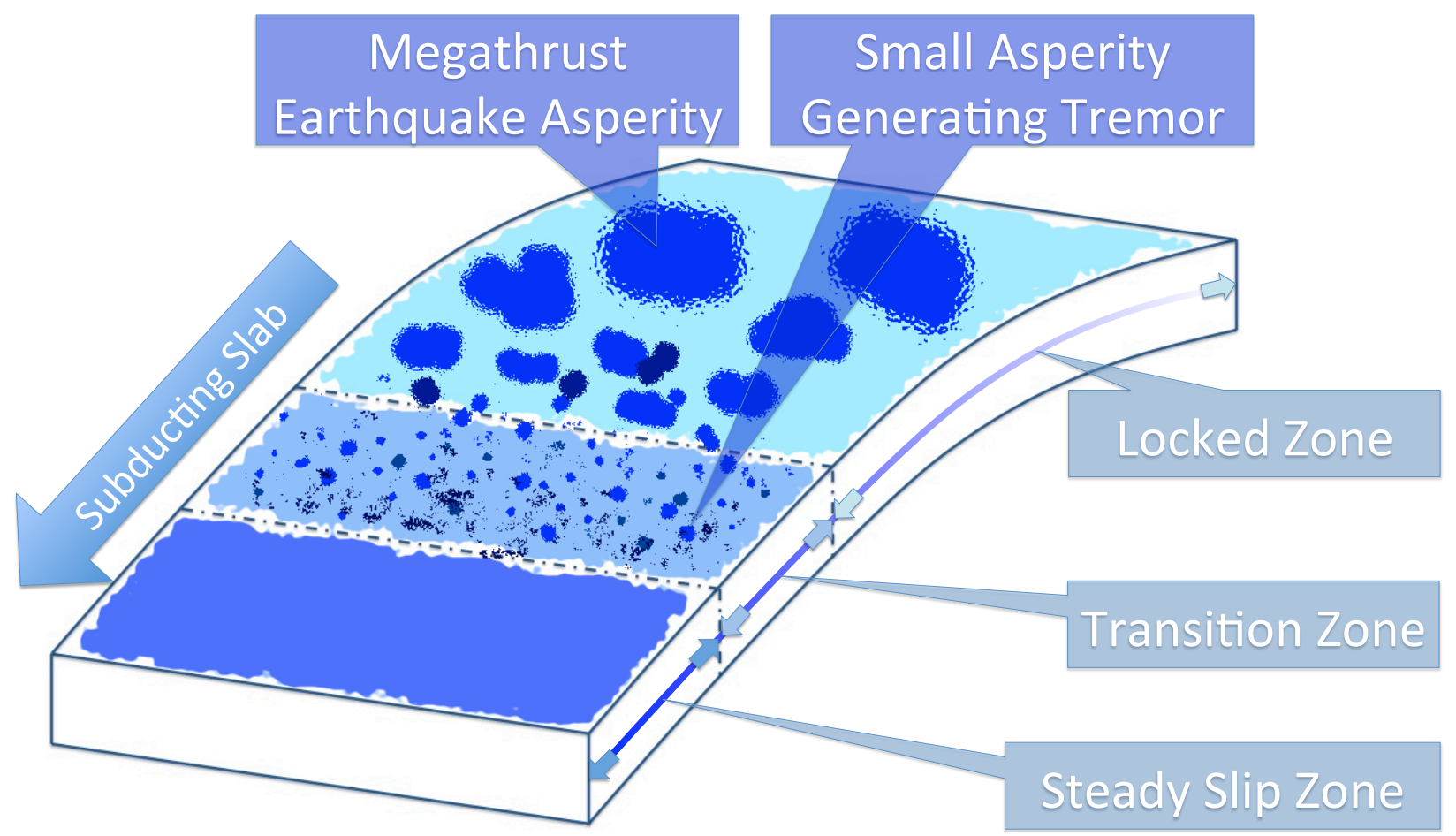

Figure 3: Conceptual model of heterogeneous seismic/aseismic transition zone of a subduction fault. Numerous small, competent asperities (darker dots) are embedded in the transition zone with more incompetent background matrix, interactions of these asperities form imbricated tremor migration patterns.

between theory and observations, we next develop specific computational realizations of the conceptual model based on rate-and-state friction.

\subsection{Rate-and-state friction models}

We consider laboratory-motivated rate-and-state friction laws (Dieterich 1979; Marone 1998; Ruina 1983). The friction coefficient $\mu$ is a function of slip velocity $V$ and a state variable $\theta$ :

$$
\mu=\mu^{*}+a \ln \frac{V}{V^{*}}+b \ln \frac{V^{*} \theta}{D_{c}}
$$

where $\mu^{*}$ is the reference steady-state friction coefficient at the arbitrary reference velocity $V^{*}, D_{c}$ is the characteristic slip distance for state evolution, $a$ and $b$ are constitutive parameters quantifying the direct and evolution effects, respectively. The evolution of the state variable $\theta$ is determined by an empirical law. We adopt here the so-called "slip law", the state evolution law that is most consistent with laboratory experiments with large velocity steps representative of the sharp slip acceleration at an SSE front (Bhattacharya and Rubin 2014):

$$
\dot{\theta}=\frac{V \theta}{D_{c}} \ln \frac{V \theta}{D_{c}}
$$

When $a-b>0$, the fault is velocity-strengthening at steady state (VS), i.e. fault friction increases as slip rate increases. On a VS fault, slip transients do not occur spontaneously, but they can be induced by external perturbations (Perfettini and Ampuero 2008). When $a-b<0$, the fault is velocity-weakening at steady state (VW), i.e. friction decreases as slip rate increases. On VW faults, spontaneous slip transients can occur if the fault stiffness is below a certain critical stiffness, e.g. a VW asperity driven by surrounding creep fails repeatedly, generating seismic events, if its size exceeds the critical size defined by:

$$
L_{c}=\frac{G D_{c}}{(b-a) \sigma}
$$



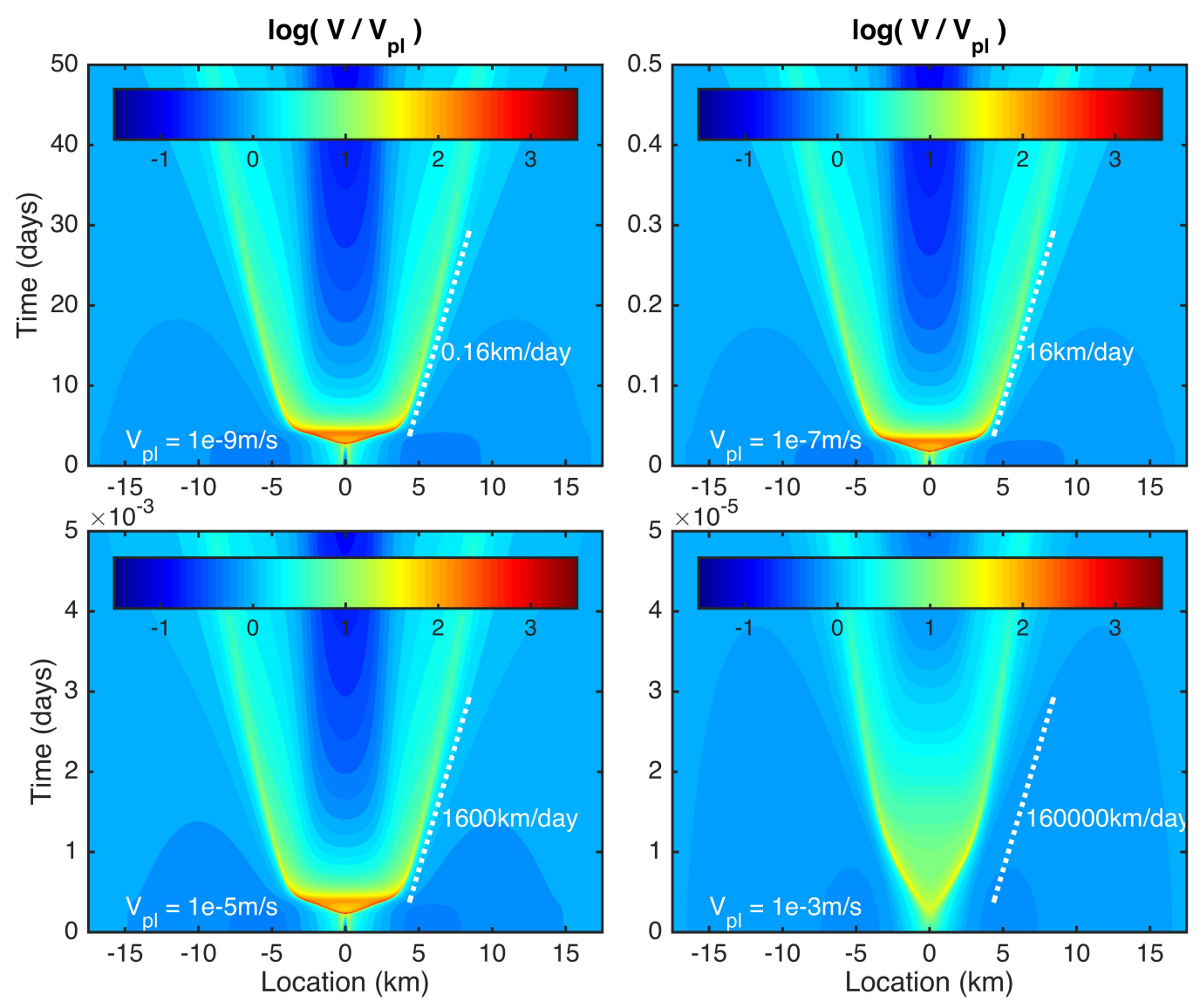

Figure 4: Comparison of the propagating velocity $V_{\text {prop }}$ of the afterslip front induced by a VW asperity surrounded by VS material, for different values of the background slip rate $V_{p l}$ labeled at the bottom left corner of each plot. The overall propagation speed is indicated in each plot by a white dashed line and a label. The results for $V_{p l}=10^{-9} \mathrm{~m} / \mathrm{s}$ to $10^{-5} \mathrm{~m} / \mathrm{s}$ indicate $V_{\text {prop }} \propto V_{p l}$. The proportionality relation degrades when intertial effects become significant, if $V_{p l}$ approaches dynamic slip rate $V_{d y n}$ and $V_{\text {prop }}$ approaches shear wave velocity (bottom-right plot).

where $\sigma$ is the effective normal stress (normal stress minus pore fluid pressure). We refer to such asperities as supercritical. Under the slip law, if an asperity is subcritical, i.e. its size is smaller than the critical size $L_{c}$, it can still be triggered by a strong enough external perturbation.

The conceptual model introduced in the previous subsection relied on a correlation between peak slip rate $V_{\max }$ and background slip rate $V_{b g}$. We can justify that assumption under the rate-and-state framework. A location on the VS fault matrix, in the vicinity of a VW asperity that just broke, experiences a positive stress perturbation $\Delta \tau$ induced by the sudden asperity slip. This perturbation is proportional to the stress drop in the asperity and decays as a function of distance from the asperity. As a result, slip in the VS area accelerates. Evaluating Equation 4, before the arrival of the afterslip front:

$$
\tau_{b g}=\sigma\left(\mu^{*}+a \ln \frac{V_{b g}}{V^{*}}+b \ln \frac{V^{*} \theta_{b g}}{D_{c}}\right)
$$

During the passage of the afterslip front, the slip first rapidly accelerates and then decelerates. The shear stress when slip rate reaches its peak $V_{\max }$ is

$$
\tau_{\text {max }}=\sigma\left(\mu^{*}+a \ln \frac{V_{\text {max }}}{V^{*}}+b \ln \frac{V^{*} \theta_{b g}}{D_{c}}\right)
$$

Here we have made the approximation $\theta \approx \theta_{b g}$, because the state variable has little time to evolve during the acceleration 

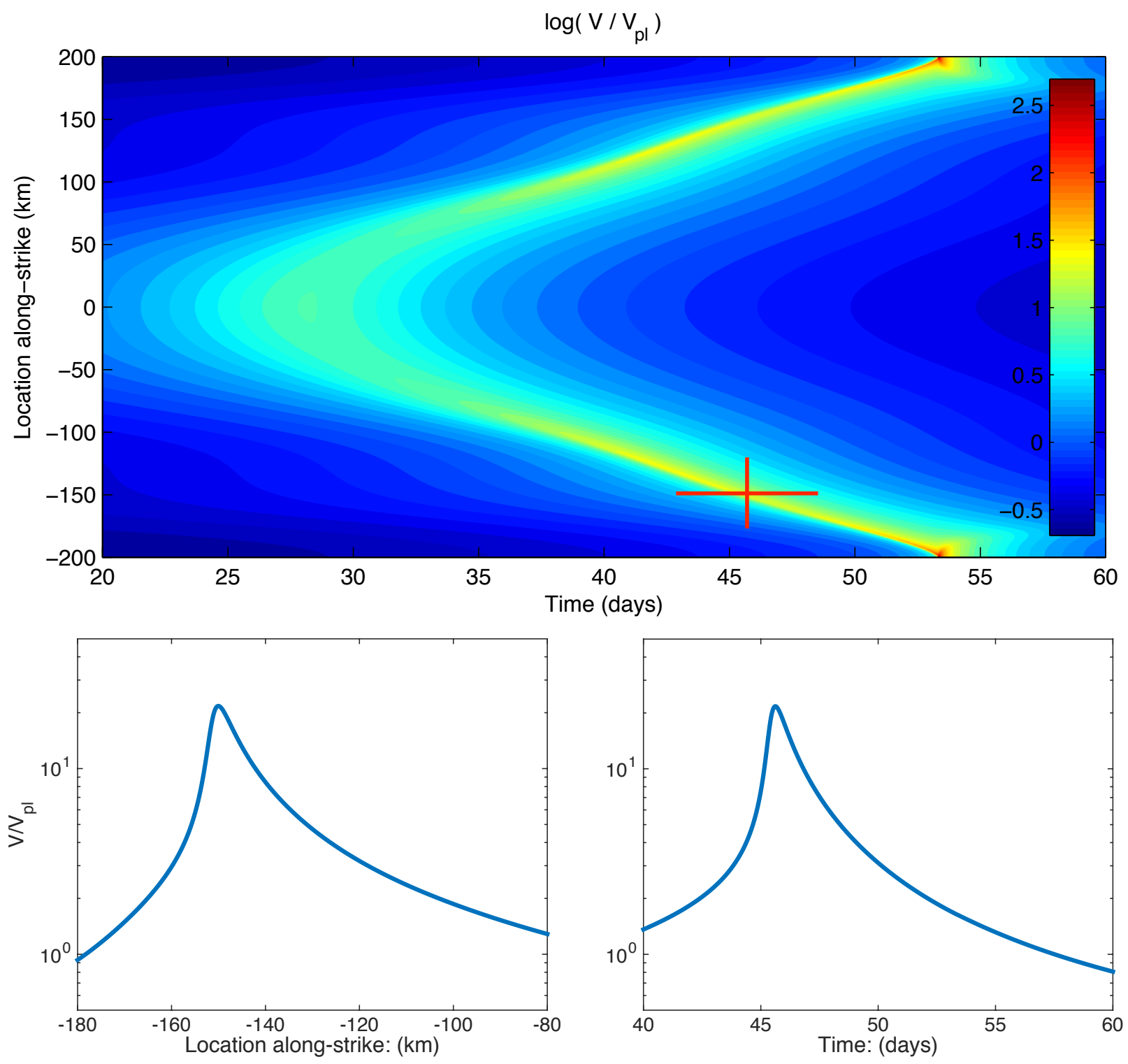

Figure 5: Example of modeled slow-slip event (SSE). Top: logarithmic slip rate normalized by plate loading rate $V_{p l}$ (colors) as a function of time and location along the fault. The SSE propagates bilaterally with a propagation velocity of about $7 \mathrm{~km} /$ day. Bottom-left: logarithmic slip rate normalized by plate velocity as a function of distance at $t=45.6$ days, marked as red cross in the upper figure. Bottom-right: logarithmic slip rate normalized by plate velocity as a function of time at $x=-150 \mathrm{~km}$, marked as red cross in the upper figure.

stage at the arrival of the afterslip front. Subtracting Equations 7 and 8 yields the stress change

$$
\Delta \tau=\tau_{\max }-\tau_{b g}=a \sigma \ln \frac{V_{\max }}{V_{b g}}
$$

We can rewrite this as

$$
V_{\max } \approx e^{\frac{\Delta \tau}{a \sigma}} V_{b g}
$$

Combining this relation with Equation 2, we get

$$
V_{\text {prop }} \propto e^{\frac{\Delta \tau}{a \sigma}} V_{b g}
$$




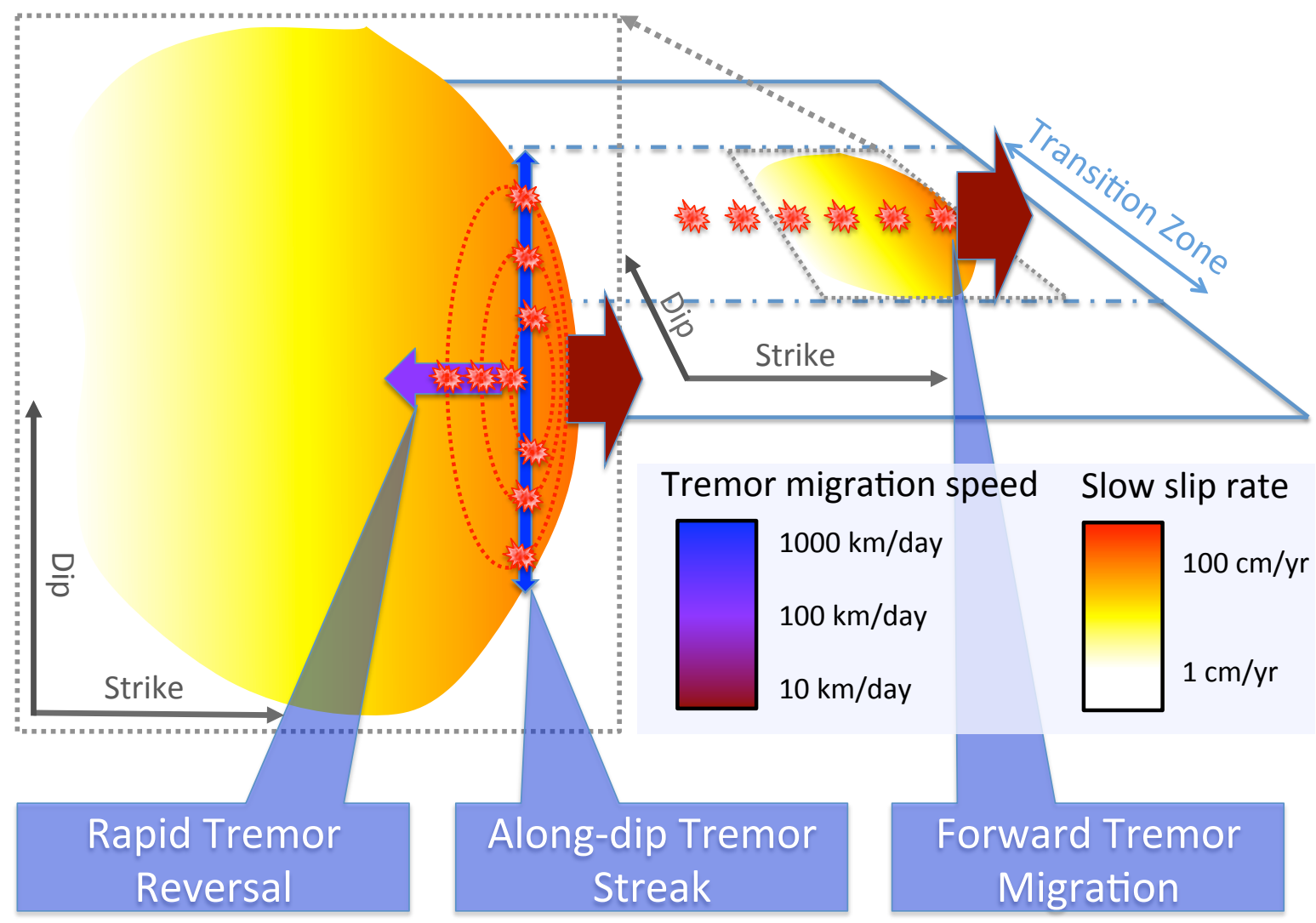

Figure 6: Conceptual model of hierarchical tremor migration patterns. Right: forward tremor migration is triggered by the on-going SSE front; yellow gradient shows the approximate slip rate distribution of SSE; red stars indicate the tremors. Left: zoom-in view. The interaction of asperities produces rapid tremor reversals and faster tremor swarms along-dip due to the mechanical correlation between migration speed and the spatial distribution of slip rate of the SSE front. Red dashed contours are isochrone contours of the post-seismic slip front induced by a breaking asperity, which propagates faster if the local background slip rate is higher.

This equation indicates that, given a spatial distribution of stress increment $\Delta \tau$ imposed by an asperity failure, the propagation speed $V_{\text {prop }}$ of the induced afterslip is proportional to the background slip velocity $V_{b g}$.

In our simulations, we adopt the quasi-dynamic approximation:

$$
\tau(x, t)=\tau^{\infty}+\frac{G}{2} H(\delta)-\frac{G}{2 C_{s}} V
$$

The first term on the right hand side $\left(\tau^{\infty}\right)$ is the external shear loading. The second term $H(\delta)$ is a linear functional representing the static stress transfer due to slip $\delta$. The inertial effects are approximated by considering the stress reduction due to the radiation of seismic waves in the direction normal to the fault plane, represented by the radiation damping term (Rice 1993), the third term (where $C_{s}$ is the shear wave velocity). These equations are solved by a numerical code based on the Boundary Element Method, QDYN (Luo et al. 2017)

In this study we consider a simplified $1.5 \mathrm{D}$ fault model with periodic boundary conditions. The original problem involves transient slip on a 2D fault region of finite width $W$, loaded by steady slip on the rest of the fault. The dimensionality of the problem is reduced by assuming the along-dip distribution of slip is fixed, so that the only remanining unknowns are the fluctuations of slip along-strike (Luo and Ampuero 2017). Other model settings are provided in section 2.3 and in appendix A2 of Luo and Ampuero (2017). We set the cell size much smaller than the 
process-zone size, $L_{b}=D_{c} G / b \sigma$, so that the cohesive zone near slip fronts and the minimum nucleation size are well-resolved in the fault matrix (Perfettini and Ampuero 2008). As our models emphasize the collective effect of interactions between asperities, the details of the spatial distribution of slip inside asperities are not essential and, to save computational resources, we represent each asperity by a single computational cell.

\subsection{SSE-driven-tremor model}

In the SSE-driven-tremor model, tremors are driven by spontaneous SSEs. The fault matrix is an unstable but aseismic material that generates SSEs spontaneously, which then drive the seismic failure of asperities (Figure 6). In order to study the multi-scale problem of SSE and RTR efficiently, in this section we first study the generation of SSEs, then the response of isolated asperities to transient loads, and finally we combine SSEs and asperities to simulate ETS.

\subsubsection{Generation of slow slip events}

SSEs can be generated by various mechanisms. Under classical rate-and-state friction, SSEs can occur in a VW strip, sandwiched between a deeper steady creep fault region and a shallower coupled seismic zone, if its along-dip width is near a critical length (e.g. Liu and Rice 2005; Rubin 2008). Another mechanism to limit slip velocity is the fault strengthening effect of gouge dilatancy in a fluid saturated fault zone (e.g. Segall et al. 2010). Slow slip can also be obtained under friction laws with two state variables (e.g. Rubin 2011) or with a transition from velocity-weakening to velocity-strengthening at increasing slip rate (e.g. Hawthorne and Rubin 2013a; Shibazaki and Iio 2003). Some of these mechanisms require tuning model parameters within a very narrow range of values, which is problematic. Because our focus is on modeling tremor migration, we conveniently adopt the SSe generation model based on friction with VW-to-VS transition. The friction coefficient $\mu$ is given by

$$
\mu(V, \theta)=\mu^{*}-a \ln \left(\frac{V_{1}}{V}+1\right)+b \ln \left(\frac{V_{2} \theta}{D_{c}+1}\right)
$$

where $V_{1}$ and $V_{2}$ are cut-off velocities of the direct effect and the evolution effect, respectively. Figure 7 shows the steady-state friction coefficient, $\mu\left(V, D_{c} / V\right)$, as a function of slip velocity, for different values of $V_{2}$. The VW-VS transition is governed by $V_{2}$ and friction properties $a$ and $b$ : it occurs at $V_{t}=V_{2} \frac{b-a}{a}$ (Hawthorne and Rubin 2013a). Strengthening effectively sets a soft limit to the maximum slip rate.

Current observations of SSE in North Cascadia provide the following constraints to our model: recurrence time is on the order of 1 year, duration is a few weeks, stress drop tens of $\mathrm{kPa}$, propagation speed about 5-10 km/day, and slip velocity about 10 to 100 times the plate velocity (Houston et al. 2011). Fault size and plate loading rate are also well constrained. Here we build up on a comprehensive study by Hawthorne and Rubin (2013a) that identified model parameters that reproduce observed SSE properties. The value of $D_{c}$ spans a relatively wide range in laboratory results and contributes to stress drop, but its effect trades off with $b$ and $\sigma$. Here, we adopt a representative laboratory value of $0.4 \mathrm{~mm}$. We explored the effect of effective normal stress $\sigma$ and cut-off velocity $V_{2}$. Figure 8 shows the peak slip rate $V_{\text {max } \_S S E}$ and propagation velocity $V_{S S E}$ measured in our simulations with various values of $\sigma$ and $V_{2}$, during periods of relatively steady SSE propagation. We exclude periods of acceleration at the end of SSEs caused by the periodic boundary conditions assumed along-strike. We find a range of parameters that reproduce the key observations quantitatively, and finally adopt a set of model parameters (Table 1) that yields SSEs with recurrence time of about 6 months, duration of about 3 weeks, propagation speed of about $7 \mathrm{~km} /$ day, slip velocity lower than $10^{-7} \mathrm{~m} / \mathrm{s}(100$ times plate velocity), and stress drop of about $10 \mathrm{kPa}$ (Figure 5).

In the model, parameter settings and resulting SSE are similar to those in the work by Hawthorne and Rubin (2013a), but there are two notable differences. We generate SSE on a fault with homogeneous friction properties. Both Hawthorne and Rubin (2013a)'s and our models have periodic boundary conditions. A trivial solution under periodic boundary condition and uniform friction properties is uniform slip, without SSE front propagation. Hawthorne and Rubin (2013a) introduced material heterogeneity, a small VS patch, to avoid convergence to the trivial uniform slip solution. Here, we obtained episodic steady-propagating SSEs in a homogeneous fault by considering heterogeneous initial conditions. In most cases, this episodic SSE behavior is a strong attractor: the fault approaches a limit cycle after a few cycles. Transient non-uniform slip solutions on homogeneous faults were previoulsy identified in rateand-state models by Horowitz and Ruina (1989). The second notable difference is that we obtain a higher ratio between peak slip velocity and propagation speed, $V_{\max \_S S E} / V_{S S E}$, than Hawthorne and Rubin (2013a). We find that increasing $V_{2}$ increases both $V_{\text {max_SSE }}$ and $V_{S S E}$, but has a stronger effect on $V_{\text {max_SSE}}$, which enables higher values 


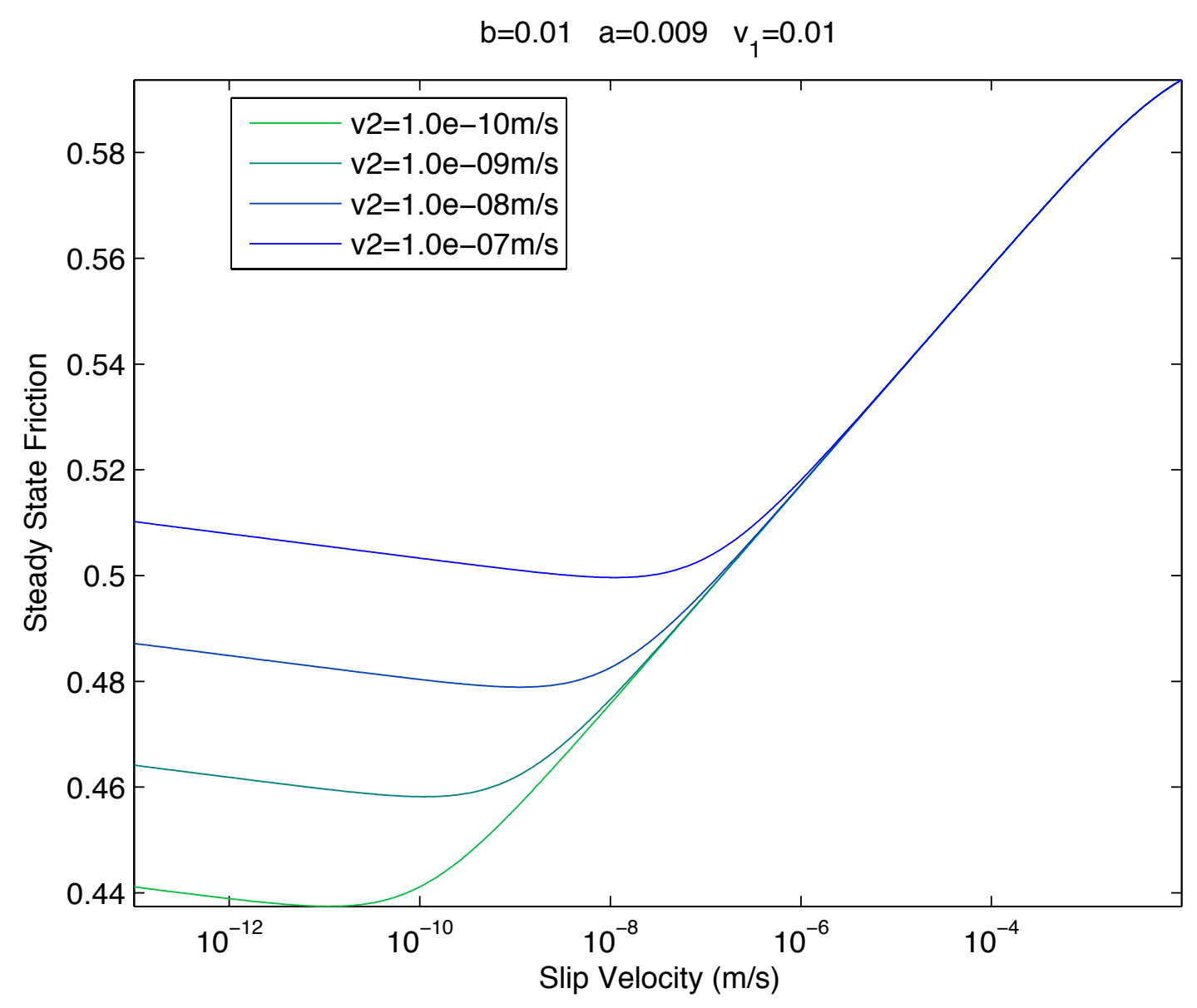

Figure 7: Steady-state friction of the rate-and state law with cut-off velocity, as a function of slip velocity, for different values of the cut-off velocity of the evolution effect $\left(V_{2}\right)$. The transition from VW to VS is controlled by $V_{2}$ if $a$ and $b$ are fixed.

of $V_{\text {max_SSE }} / V_{S S E}$ (Figure 8). We will find this useful to obtain ratios between RTR and SSE propagation speeds as high as observed in Cascadia (section 4.4), a goal that has proven challenging for previous models (e.g. Ando et al. 2010, 2012; Colella et al. 2011, 2013; Hawthorne and Rubin 2013b).

\subsubsection{Response of isolated asperities to transient loading}

Basic tremor observations and our conceptual model (section 2.1) provide constraints on asperity properties. Asperities should be pure VW (no VW-VS transition) so that they fail with high slip rates associated to seismic radiation, i.e. exceeding the dynamic velocity $V_{d y n}=2(a-b) C_{s} \sigma / G$ above which radiation damping becomes important (Rubin and Ampuero 2005). Because our focus is on ETS, to limit the computational cost of the simulations we consider only subcritical asperities, so that failure is induced during SSEs but does not occur spontaneously in between SSEs. Moreover, in Cascadia the "background tremor" activity in between ETS events is weaker and deeper (Wech et al. 2010). Lastly, we focus on modeling asperities that are triggered only a few times by an SSE. For a given loading rate, an asperity that breaks infrequently has a higher stress drop than an asperity that breaks often. It hence generates a stronger afterslip transient, which favors the emergence of migrating swarms. Observations show that LFEs break multiple times during a large SSE, but often in bursts that have been associated to secondary (smaller-scale) slip transients (e.g. Lengliné et al. 2017; Peng et al. 2015).

To identify values of asperity properties that allow asperities to be triggered only a few times by an SSE, we study the response of an isolated asperity to a transient loading akin to the loading imposed by the passage of a SSE. We 

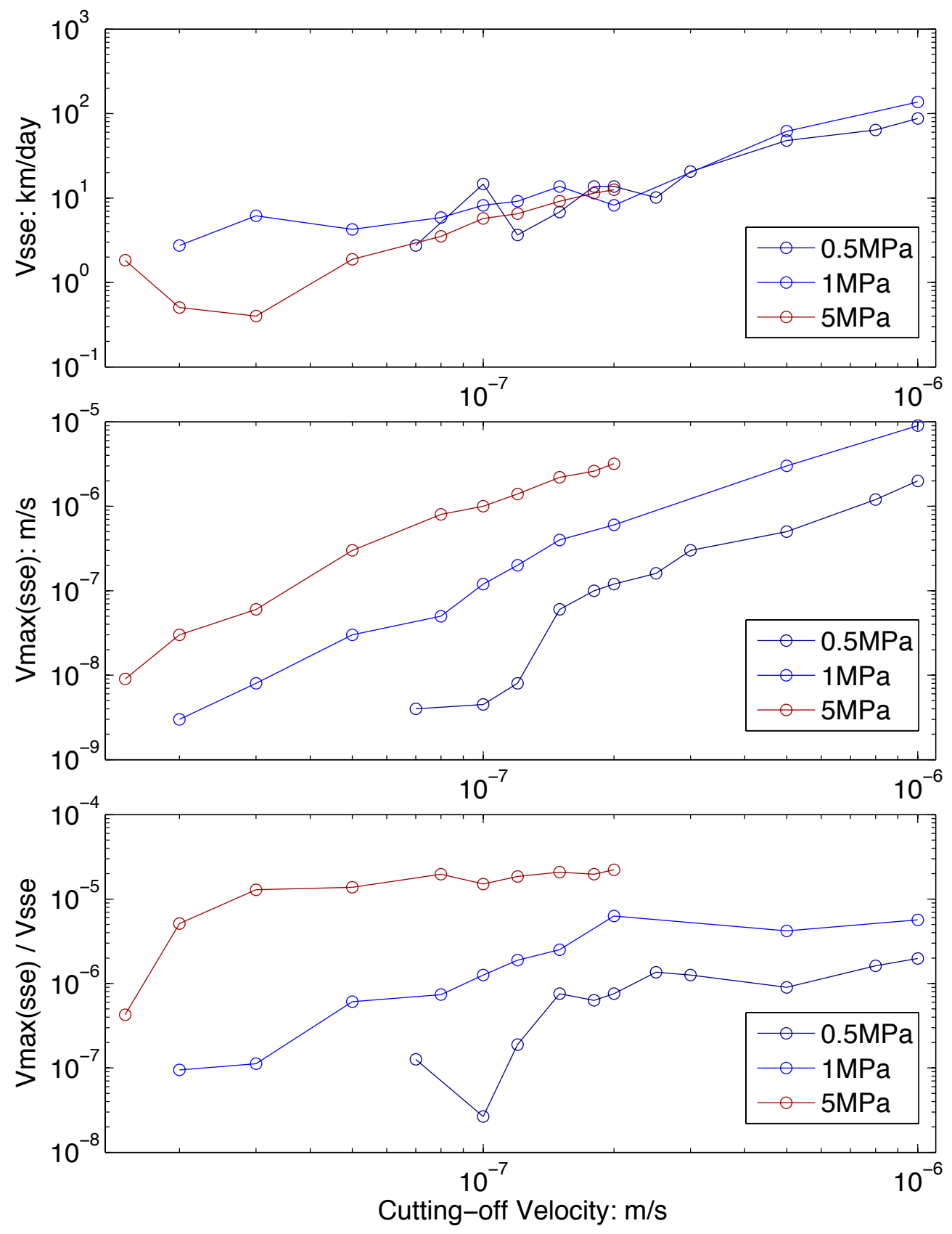

Figure 8: Propagation velocity of SSE ( $\left.V_{S S E}\right)$ and peak slip rate inside the SSE front $\left(V_{\text {max }} S S E\right)$ as a function of the cut-off velocity $V_{2}$, for three different values of effective normal stress $\sigma$. Within a certain range of $V_{2}$, both $V_{S E E}$ and $V_{\text {max_SSE }}$ increase with increasing $V_{2}$. However, $V_{\text {max }} S S E$ increases faster, leading to large $V_{\text {max_SSE }} / V_{S E E}$ ratios and ultimately larger $V_{R T R} / V_{S S E}$ ratio.

consider an elementary model: we study the response of a single-degree-of-freedom spring-block system to Gaussian shaped transient loadings as a function of the loading duration $T_{p}$, which represents the SSE rise time (local slip duration). (Figure 9) shows the resulting slip velocity for a range of $T_{p}$ values. We find that the asperity is triggered only once if $T_{p}$ is comparable to $T_{\text {rec_asp }}=\frac{2 D_{c}}{V_{p l}} \sqrt{\frac{a}{b-a}}$, the recurrence time of slip on an asperity of critical size. If $T_{p} \ll T_{r e c_{-} a s p}$ the asperity is not triggered, and if $T_{p} \gg T_{r e c_{-} a s p}$ it breaks too many times (when applying a higher transient loading amplitude than shown in Figure 9). Henceforth we consider subcritical asperities with $T_{p} \approx T_{\text {rec_asp }}$. 


\begin{tabular}{|c|c|}
\hline Physical properties & Value \\
\hline fault period $L$ & $400 \mathrm{~km}$ \\
\hline fault width $W$ & $110 \mathrm{~km}$ \\
\hline shear modulus $G$ & $30 G P a$ \\
\hline shear wave velocity $C_{s}$ & $3000 \mathrm{~m} / \mathrm{s}$ \\
\hline reference friction coefficient $\mu^{*}$ & 0.6 \\
\hline tectonic loading rate $V_{p l}$ & $10^{-9} \mathrm{~m} / \mathrm{s}$ \\
\hline \multirow[t]{2}{*}{ asperity actual size $L_{a s p}$} & $100 m($ typical $)$ \\
\hline & $31.25 m$ to $400 m$ (various) \\
\hline \multirow[t]{2}{*}{$b \sigma_{b g}$} & $10 \mathrm{KPa}($ typical $)$ \\
\hline & 5 to $50 \mathrm{KPa}$ (various) \\
\hline \multirow[t]{2}{*}{$b \sigma_{a s p}$} & 100 to $500 \mathrm{KPa}$ (typical) \\
\hline & 10 to $1000 \mathrm{KPa}$ (various) \\
\hline \multirow[t]{2}{*}{$a \sigma_{b g} @$ SSE-driven model } & $9 \mathrm{KPa}($ typical $)$ \\
\hline & 4.5 to $45 \mathrm{KPa}$ (various) \\
\hline \multirow[t]{2}{*}{$a \sigma_{b g} @$ tremor-driven model } & $12 \mathrm{KPa}($ typical $)$ \\
\hline & 6 to $60 \mathrm{KPa}$ (various) \\
\hline \multirow[t]{2}{*}{$a \sigma_{a s p}$} & 90 to $450 \mathrm{KPa}$ (typical) \\
\hline & 9 to $900 \mathrm{KPa}$ (various) \\
\hline \multirow{2}{*}{$\begin{array}{l}\text { background characteristic slip distance } D_{c_{-} b g} \\
\text { asperity characteristic slip distance } D_{c_{-} a s p}\end{array}$} & $4 \times 10^{-4} \mathrm{~m}$ \\
\hline & $\begin{array}{l}1 \times 10^{-4} \text { to } 1 \times 10^{-3} \mathrm{~m} \text { (typical) } \\
4 \times 10^{-5} \text { to } 4 \times 10^{-3} \mathrm{~m} \text { (various) }\end{array}$ \\
\hline \multirow{4}{*}{$\begin{array}{l}\text { cut-off velocity (direct effect) } V_{1} @ \text { SSE-driven model } \\
\text { cut-off velocity (indirect effect) } V_{2} @ \text { SSE-driven model } \\
\text { asperity density }\end{array}$} & $0.01 \mathrm{~m} / \mathrm{s}$ \\
\hline & $10^{-8}$ to $10^{-5} \mathrm{~m} / \mathrm{s}$ (various) \\
\hline & 10 to $20 \%$ (typical) \\
\hline & 5 to $50 \%$ (various) \\
\hline
\end{tabular}

Table 1: Typical parametric settings of rate-and-state simulation

\subsubsection{Modeling forward tremor migration and RTRs}

To combine the above models of SSE and asperities, we first simulate multiple SSE "warm-up cycles" without asperities using the parametric settings identified in section 2.3.1 until the fault behavior reaches a limit cycle independent of the initial conditions. We then add asperities and run a few more warm-up cycles. The asperities are evenly distributed in space, with the properties identified in the previous section. We explore various values of asperity spacing and relative strength defined as the contrast of $|b-a| \sigma$ values inside and outside asperities. We assign random $D_{c}$ values to each asperity. This results in a briad range of individual asperity criticalness defined as $\beta=L_{\text {asp }} / L_{c_{-} a s p}$, where $L_{\text {asp }}$ is the asperity size (a single cell) and $L_{c_{\_} a s p}$ is the critical size defined in Equation 6. Detailed parameter settings are presented in Table 1.

We find that, although the rich SSE and tremor behavior can be reproduced with a relatively wide range of asperity densities and relative strengths, the highly heterogeneous asperity criticalness is essential to generate RTRs matching observations in Cascadia. Thus we hypothesize that heterogeneity of asperity properties is a necessary condition for the generation of RTRs (section 3.3).

\subsection{Tremor-driven-SSE model}

In the process of our exploration of the SSE-driven-tremor model, we found that asperities can have a large impact on the overall behavior of the fault. We thus conducted an extensive numerical and theoretical study of the stability of faults with mixtures of VW and VS materials (Luo and Ampuero 2017). That study complements previous work by Dublanchet et al. (2013), Skarbek et al. (2012), and Yabe and Ide (2017). The results show that a composite fault can be unstable even if the VW material occupies only a small fraction of the fault surface, provided it has high enough strength contrast relative to the VS material, quantified by the ratio of $|b-a| \sigma$ between the two materials. In the SSE and tremor environment, strength contrast between asperities and matrix is likely due to contrast of effective normal stress $\sigma$ arising from spatial and temporal fluctuations of fault zone fluid pressure. 

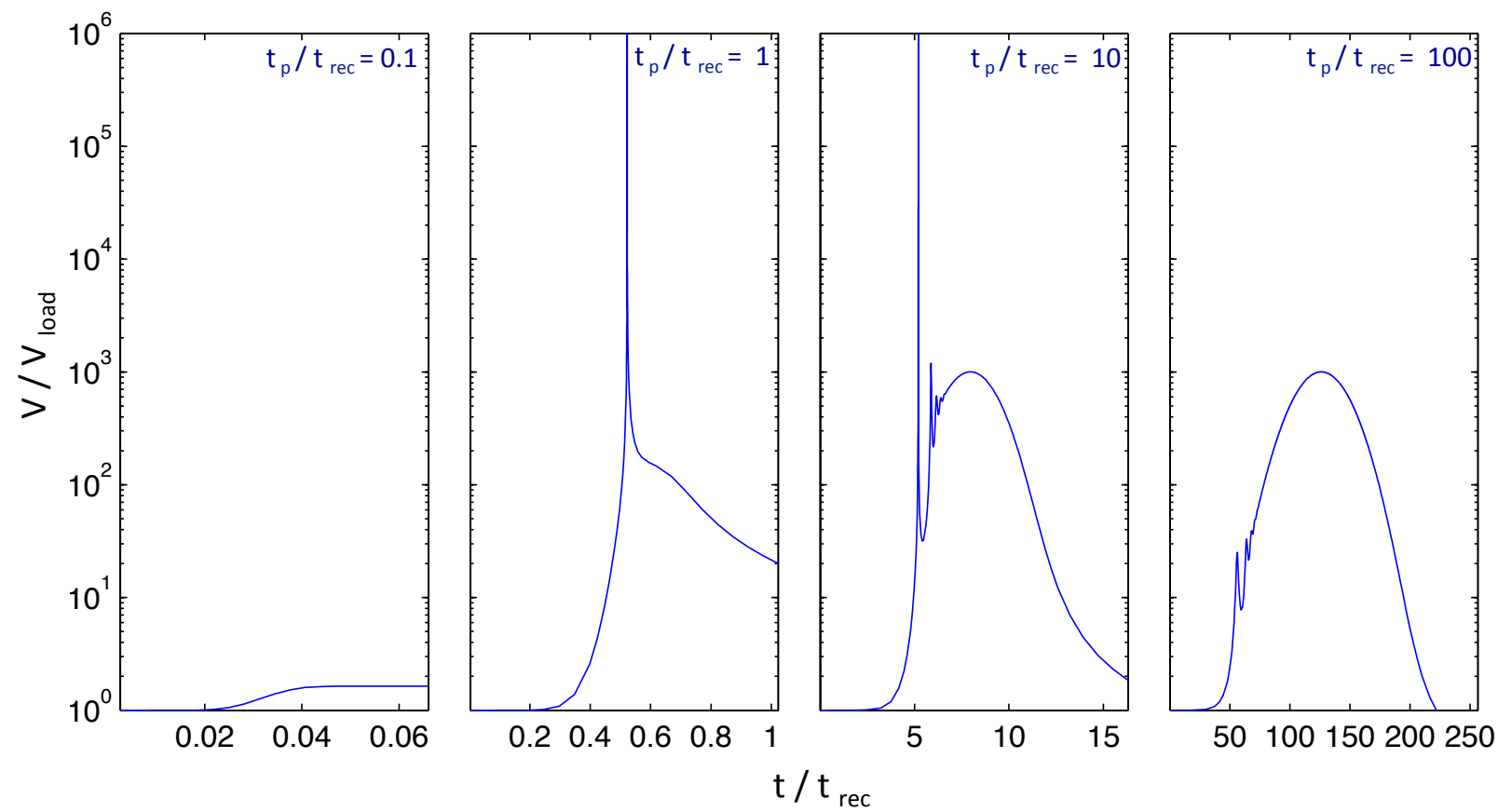

Figure 9: Slip velocity of a sub-critical spring-block system in response to transient loading with four different durations (indicated by labels at the top-right corner of each plot). The simulation time is normalized by the characteristic recurrence time $\left(T_{\text {rec_asp }}\right)$ of a critical asperity. Slip rate is normalized by the background loading rate $V_{p l}$. The amplitude of the imposed loading perturbation is 1000 times $V_{p l}$. From left to right, the duration of the perturbation $\left(T_{p}\right)$ is $0.1,1,10$ and 100 times $T_{r e c \_a s p}$, respectively. If $T_{p}$ is comparable to $T_{r e c \_a s p}$, the system breaks seismically and only once during the loading transient.

The study of the stability of composite faults (Luo and Ampuero 2017) suggests that the mixture of VW and VS materials with relative strength contrast is a sufficient ingredient to reproduce a broad spectrum of fault behavior ranging from slow to rapid transients. In particular, it provides insight on the material mixture properties leading to spontaneous slip transients on heterogeneous faults. This motivates us to develop an alternative model which, unlike the SSE-driven-tremor model, can generate SSEs and tremors without appealing to friction with VW-VS transition in the fault matrix. In our tremor-driven-SSE model friction in the matrix is purely VS. The asperity properties are similar to those in the SSE-driven-tremor model. A distinct feature of the tremor-driven-SSE model is that SSEs are generated only in the presence of asperities; they emerge as the collective behavior of interacting VW and VS patches.

The SSE-driven-tremor model and tremor-driven-SSE model are end-member models to address the first key question formulated in section 1: is SSE a cause or a result of tremor? The considerations on asperity properties developed for the SSE-driven-tremor model are also valid for the tremor-driven-SSE model, if the loading on each asperity is viewed as imposed by the average slip rate resulting from the neighboring asperity failures and their afterslip.

\section{Results}

\subsection{Slow-slip events}

Both SSE-driven-tremor model and tremor-driven-SSE model can reproduce SSEs in quantitative agreement with observations in Cascadia (Figure 5). The target SSE properties include recurrence time of about 6 months, duration of about 3 weeks, propagation speed of about $7 \mathrm{~km} /$ day, slip velocity lower than $10^{-7} \mathrm{~m} / \mathrm{s}$ (100 times plate velocity), stress drop of about $10 \mathrm{kPa}$, and a spatial span over $100 \mathrm{~km}$. These SSE properties can be modified to match observations 
in other regions by varying parameters like fault dimension, effective normal stress and frictional properties. In the SSE-driven-tremor model, SSE properties are mainly controlled by effective normal stress $\sigma$ and cut-off velocity $V_{2}$. They remain mostly unchanged by the addition of asperities, unless their density or relative strength are too high.

In the tremor-driven-SSE model there is no SSE in the absence of asperities, the SSE is rather the combined result of the post-seismic slip transients of asperities. Luo and Ampuero (2017) show how to predict the stability of slip on a composite fault with a regular alternation of VW and VS segments. Their findings suggest that in order to generate spontaneous slip transients the overall (homogenized) fault should be velocity weakening. Applying their results to the tremor-driven-SSE model, we infer that the overall relative strength (ratio between $|b-a| \sigma$ values of asperity and matrix) should exceed a certain value. With fixed asperity density, the larger the strength contrast is, the faster the transient slip rate will be, ranging from SSE to seismic behavior. In a homogeneous pure VW fault with the slip law, SSEs occur only within a narrow range of fault width over critical length $W / L_{c}$ (Rubin 2008). In our simulations of pure VW faults, the range of fault width $\mathrm{W}$ permitting steady SSE propagation over long distances is even narrower than the range that permits episodic aseismic transients. However, we find that, with the addition of asperities, the range of W permitting realistic SSEs is much wider than in a homogeneous VW fault, and no fine-tuning is required. We hypothesize that this behavior is enabled by a broad range of individual criticalness of asperities and by the non-linear nature of rate-and-state friction.

\subsection{Tremor activity featuring rapid tremor reversals}

The RTRs observed in Cascadia have a propagation distance of about $20 \mathrm{~km}$ and travel about 5 to 50 times faster than the large-scale forward tremor migration. Varying the spatial distribution and frictional properties of asperities, our models generate various tremor and SSE phenomena that are in qualitative agreement with observations in Cascadia.

In the SSE-driven-tremor model, we systematically studied the effect of key model parameters. We varied $b \sigma$ inside asperities from $10 \mathrm{kPa}$ to $1 \mathrm{MPa}$, which is 1 to 100 times the background value outside the asperities. We varied $D_{c}$ inside the asperities from $4 \times 10^{-5} \mathrm{~m}$ to $4 \times 10^{-3} \mathrm{~m}, 0.1$ to 10 times the background value. We also varied the distance between asperities from 1 to about 20 cells, that is, 1 to 20 times the size of a single-cell asperity. We varied these parameters while keeping the asperity individual criticalness $\beta$ in a typical range of 0.01 to 0.6 . We successfully simulated tremor migration patterns in quantitative agreement with observations in Cascadia (Figure 10): recurrence interval of ETS, spatial-temporal distribution of tremor migrations, forward and reverse tremor migration speed (and their ratio). The slow forward tremor propagation is naturally associated with sequential asperity triggering by a propagating SSE. Less trivially, the model produces RTRs similar to those observed in Cascadia: spatially scattered swarms back-propagating at fast speed, $V_{R T R}$, about one order of magnitude faster than the forward migration speed, $V_{S S E}$, with spatial distribution patterns resembling observations in Cascadia (Houston et al. 2011) and propagation distances around $10 \mathrm{~km}$. The $V_{R T R} / V_{S S E}$ ratio spans a moderately broad range of values, owing to the randomness of asperity properties and to the non-linear nature of the model. In the example shown in (Figure 11) $V_{R T R} / V_{S S E}$ ranges from 2 to 10, with an average value around 5, overlapping with the lower end of values observed in Cascadia. Varying the value of the cutoff velocity $V_{2}$ we achieve more realistic values up to $V_{R T R} / V_{S S E} \approx 20$ (Figure 12). In the analysis of our simulation results, we define tremors as asperity failure events with peak slip velocity exceeding a certain threshold (e.g. $1 \mathrm{~mm} / \mathrm{s})$. More tremors are detected if we lower the velocity threshold. The range of model parameters that produce realistic results is relatively large: e.g. with an asperity density of $20 \%$, models with relative strength ranging from 10 to 50 reproduce hierarchical tremor behaviors in quantitative match with observations.

The tremor-driven-SSE model, with parameter settings similar to the SSE-driven-tremor model, can also simulate the observed tremor migration patterns in quantitative agreement with observations in Cascadia (Figure 13). In this model, the forward tremor migration operates by a progressive cascade of asperity failures mediated by their intervening aseismic afterslip. RTRs also result from a cascade process, but propagate much faster due to the elevated background slip rate owing to previous asperity activity. RTRs in the tremor-driven-SSE model have a wider range of propagation speeds than in the SSE-driven-tremor model. The $V_{R T R} / V_{S S E}$ ratio can be as high as 50, in agreement with the range of values observed in Cascadia of $V_{R T R} / V_{S S E} \approx 5$ to 50 . From the perspective of $V_{R T R} / V_{S S E}$ ratios, the tremor-driven-SSE model outperforms the SSE-driven-tremor model

Other observed tremor migration patterns, including tremor halting and branching (slower reversals), acceleration and deceleration (Kao et al. 2009), are also reproduced by both models. Figure 14 shows an example of modeled tremor halting and branching phenomena, where tremor propagation stops for hours, then resumes and branches. 


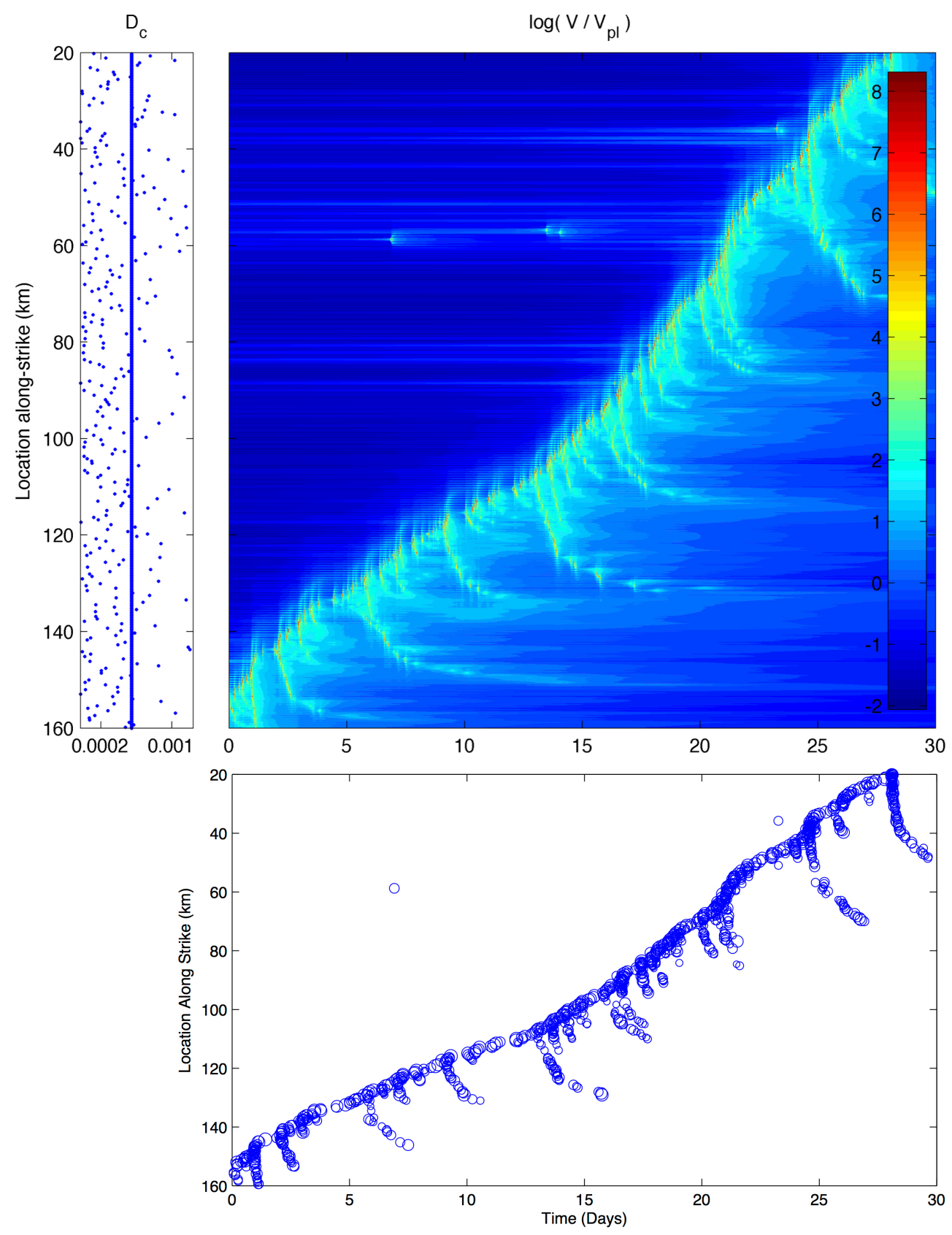

Figure 10: Top: modeled tremor forward migration and RTRs (rapid tremor reversals). Colors show the logarithmic slip rate normalized by plate loading rate $V_{p l}$. The left panel indicates the characteristic slip distance $\left(D_{c}\right)$ of the corresponding point on the fault. Every point with different $D_{c}$ values are VW asperities (i.e. no velocity weakening to strengthening transition) and have a much higher $(b-a) \sigma$ than the background. Bottom: seismicity distribution, each circle indicates a seismic event (slip velocity larger than $! \mathrm{mm} / \mathrm{s}$ ) and its size is scaled with the magnitude.

\subsection{Nucleation of Rapid Tremor Reversals}

We found that the characteristic slip distance $D_{c}$ of the asperities plays a very important role in tremor migration patterns. For instance, RTRs occur rarely in our models because they nucleate at the asperities with largest $D_{c}$ (Figure 15). We hypothesize that since these asperities are relatively strong, they do not break immediately at the arrival of the slow slip front, but are delayed by a period that depends on their relative stiffness. The delay allows neighboring 

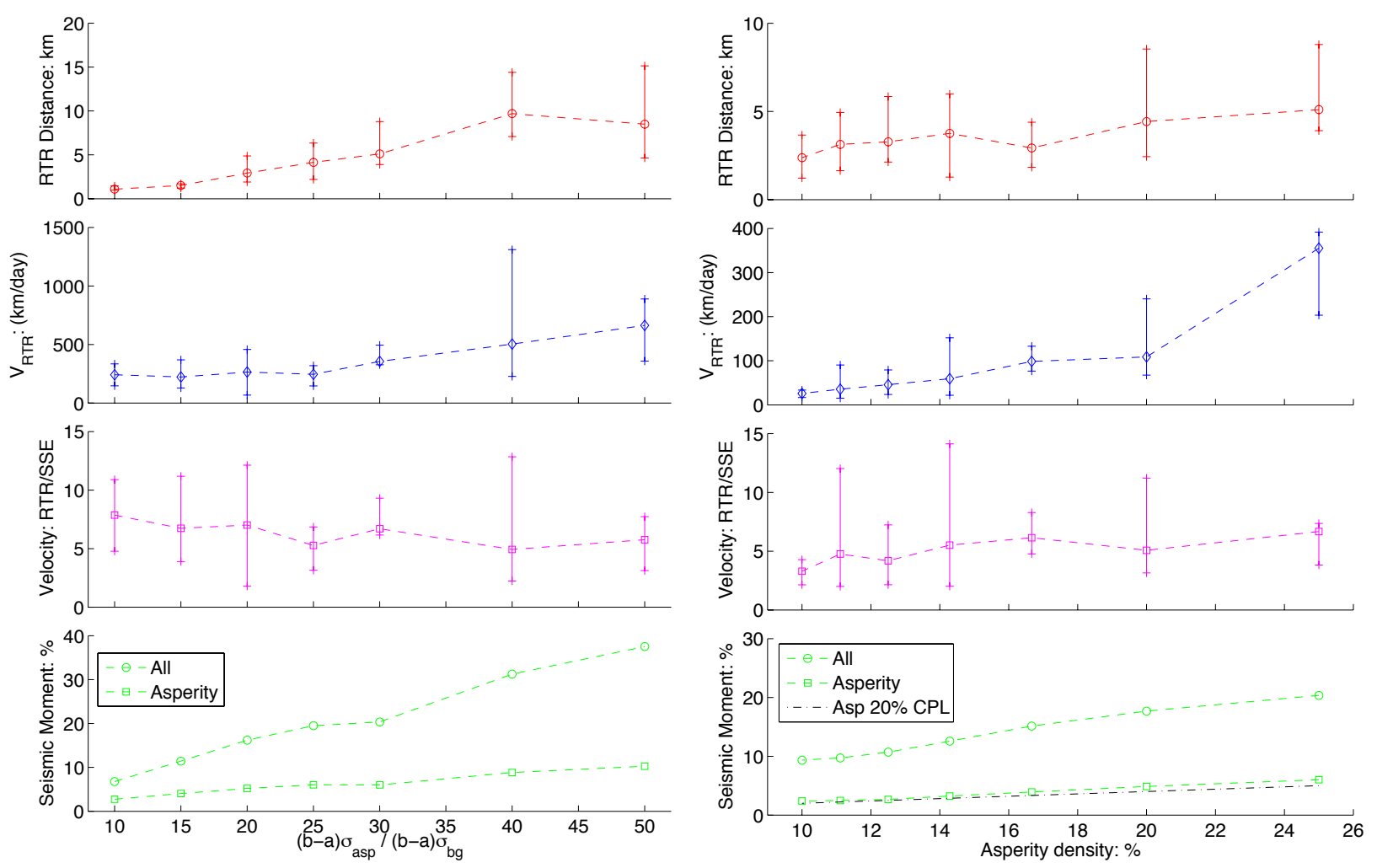

Figure 11: Effect of strength contrast (left) and density of asperities (right) on tremor properties. The strength contrast is quantified by the ratio of $|b-a| \sigma$ inside and outside asperities. From top to bottom: RTR propagating distance, RTR propagation speed, ratio of $V_{R T R} / V_{S S E}$. Vertical bars show the range. Both RTR propagating distance and velocity increase with increasing $b \sigma$ or asperity density, while $V_{R T R} / V_{S S E}$ is not strongly affected by either values. Bottom: ratio of moment released seismically to total moment released during a tremor episode, for the whole fault (circles) and for asperities only (squares). Only a small fraction of the moment is released seismically. The dashed line indicates a coupling ratio of all the asperities of $20 \%$.

asperities to recover their strength and become again capable of triggering strong enough afterslip to sustain a cascading RTR. To confirm this idea, we designed a pair of controlled numerical experiments as shown in Figure 16. In the first simulation we set 10 very strong asperities with $D_{c}$ value about one order of magnitude larger than that of the remaining asperities. All the resulting RTRs nucleated from these strong asperities. In the next simulation we removed three of the strong asperities (indicated by orange blocks in Figure 16). The RTRs that nucleated from those three asperities in the first simulation disappeared in the second simulation. Moreover, the other reversals that were either stopped or slowed down by these strong asperities in the first simulation, propagated faster and further beyond these removed asperity locations in the second simulation. The comparison results show that strong asperities not only nucleate RTRs but can also act as barriers that slow down or even stop RTRs.

Tremor halting and branching occurs by the same mechanism but on asperities with even larger $D_{c}$ (Figure 14) (either being a single asperity of very high $D_{c}$ value, or a group of neighboring asperities with high $D_{c}$ ). These very strong asperities induce longer delays before breaking, until the SSE slip velocity drops significantly. The low background slip velocity reduces the propagating speed of the subsequent post-seismic slip, resulting in a very slow tremor reversal which appears as a tremor halting and branching episode.

\subsection{Effects of asperity properties on tremor migration speeds and seismic coupling}

Further study reveals that frictional properties inside the asperities control the RTR migration distance and speed, as well as the seismic coupling defined as the ratio of seismic moment to total moment (Figure 11).

Increasing the strength contrast (ratio of $|b-a| \sigma$ inside and outside the asperities), while varying the asperities' 


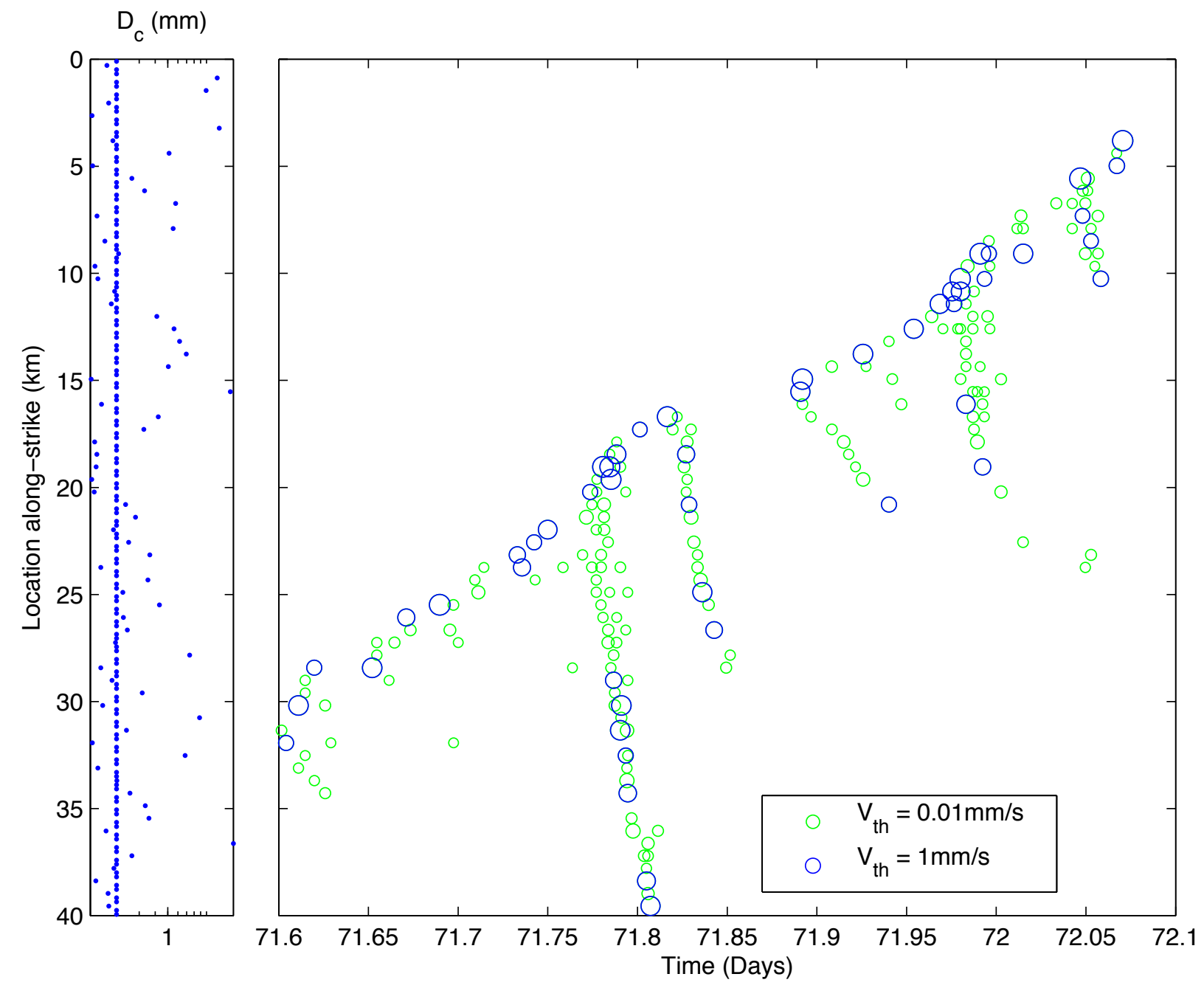

Figure 12: Higher $V_{R T R} / V_{S S E}$ ratio, analogous to seismicity plot in Figure 10, achieved by tuning the cut-off velocity $V_{2}$. Green circles show the tremor events detected with a much lower velocity threshold. Note that more tremor activities are detected with the lower threshold, including RTR slowdown near day 72.

$D_{c}$ value to preserve the asperity criticalness, has very similar effects as decreasing the distance between asperities. In particular, both increase the propagation distance reached by RTRs and their migration speed. In contrast, the ratio $V_{R T R} / V_{S S E}$ shows no strong correlation with these asperity properties. In addition, if the asperity distribution is too dense or the strength contrast is too large, the whole fault becomes seismic for both SSE-driven-tremor and tremor-driven-SSE model. If the asperity distribution is too sparse or the relative strength is too large, the interaction between asperities is too weak to produce RTRs in the SSE-driven-tremor model, and the whole fault creeps without transients in the tremor-driven-SSE model as in a sub-critical VW fault with $W<L_{c}$. These findings are consistent with those of the basic study of composite faults by Luo and Ampuero (2017).

In our models, the moment released seismically by asperities is only a small fraction, about $5 \%$ to $20 \%$ in most cases, of the total moment released by ETS events. Here we defined as seismic the moment cumulated while the local slip rate is higher than $V_{d y n} \approx 1 \mathrm{~mm} / \mathrm{s}$, and the total moment as the moment cumulated between the first time fault slip rate at any point on the fault reaches $1 \mathrm{~mm} / \mathrm{s}$ and the last time every point of the fault drops below $1 \mathrm{~mm} / \mathrm{s}$ (Figure 11). The range of seismic coupling values is small, but at least one order of magnitude larger than observations. Kao et al. (2010) suggested the seismic coupling is of the order of $0.1 \%$ or less. Note that observational studies report a frequency-limited measure of the moment of tremor or LFE, which most likely represents a lower bound of the real tremor moment. It is also worth noting that in the tremor-driven-SSE model, despite being driven by asperity failures, 


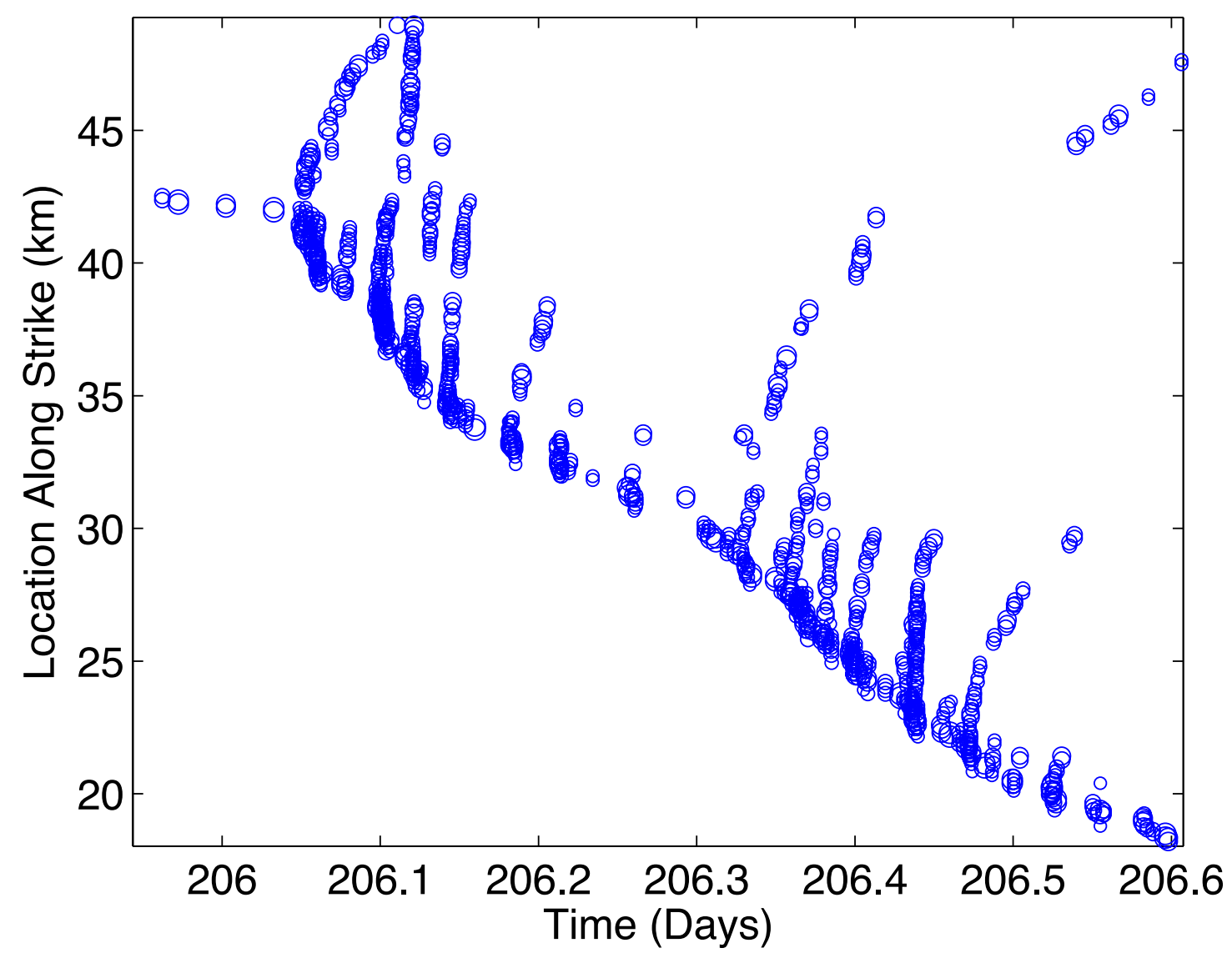

Figure 13: Tremor-driven-SSE model simulation. Various tremor migration patterns are also observed in this model. Figure analogous to Figure 10.

most of the moment of ETS is aseismic, in the form of afterslip induced by asperity failure. We hypothesize that low seismic coupling is due to two reasons: the majority of the fault consists of VS materials and all the VW asperities are individually subcritical.

\section{Discussion}

\subsection{Comparison of SSE-driven-tremor and tremor-driven-SSE models}

The two end-member models developed here have their own strengths and weaknesses. The SSE-driven-tremor model requires a more sophisticated friction law, whose experimental support is limited, but allows SSEs without tremor, which has been reported in natural faults (e.g. SSE in New Zealand, Peng and Gomberg (2010)). The tremor-drivenSSE model is based on a more conventional friction law and matches certain observations better and without fine tuning, in particular the ratio $V_{R T R} / V_{S S E}$. However it cannot explain observations of SSE without tremor, unless the fault is in a near-critical state in which asperity failures are too slow to be detected seismologically but strong enough to sustain an SSE.

We can identify potentially observable characteristics of the two models that can help distinguish them. Seismic coupling is not a discriminating characteristic. Given the small seismic moment attributed to tremors (Kao et al. 2010), the SSE-driven-tremor model is a widely accepted concept. However, as reported in section 3.4, in the tremor-drivenSSE model the fraction of moment released seismically is also small. A key difference between these two models is 


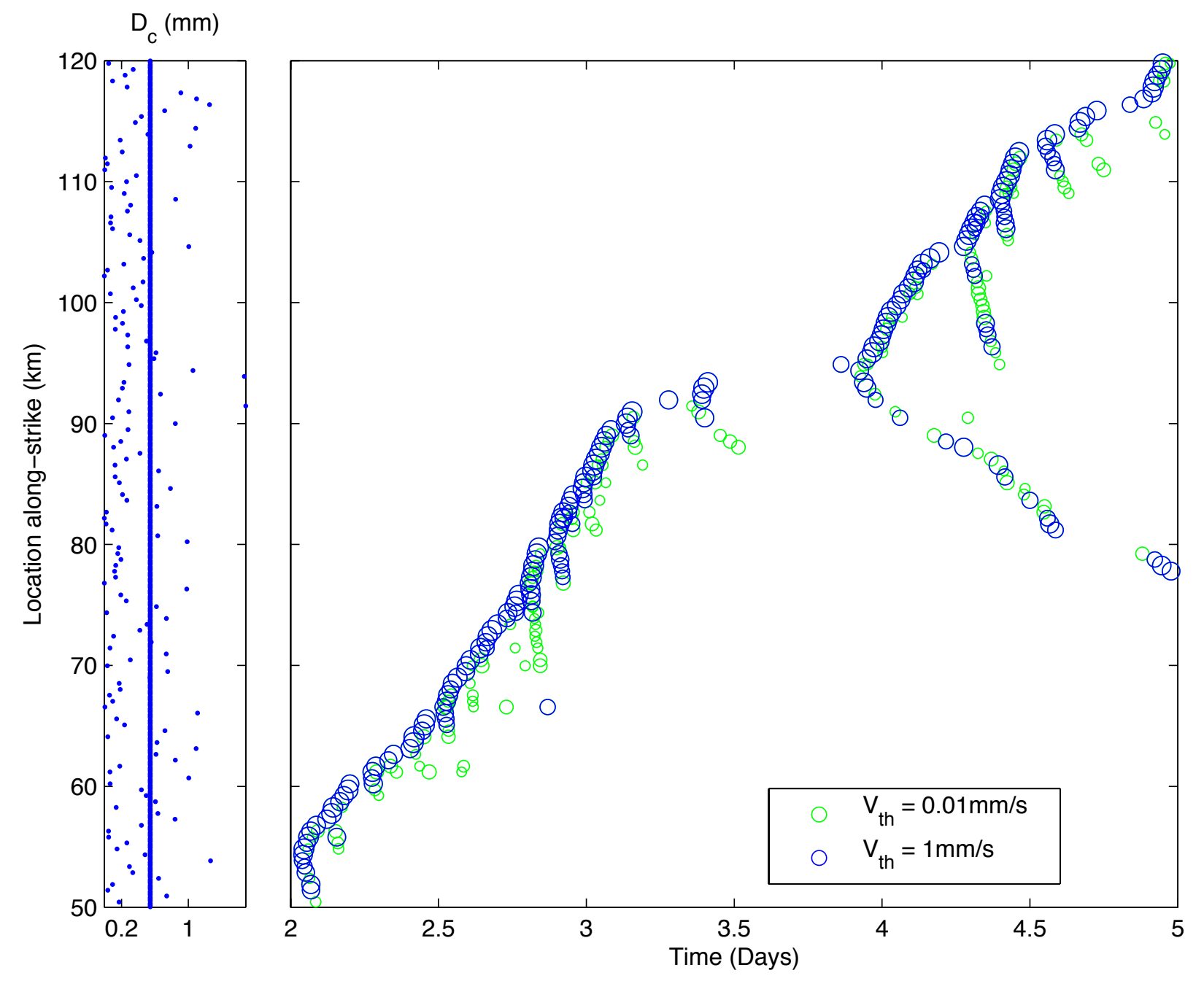

Figure 14: Tremor halting and branching. Tremor propagation halts for hours, and then resumes and branches. Different color in the seismicity plot shows results using different detection thresholds.

the asperity recurrence pattern. The stacked inter-event time between failures of a same asperity (normalized by the mean inter-event time) decays as a function of time $t$ after its first break as $1 / \sqrt{t}$ in the SSE-driven-tremor model and as $1 / t$ in the tremor-driven-SSE model (Figure 17). An analysis of LFEs in the Mexico and Cascadia subduction zones by Lengliné et al. (2017) reveals a 1/t decay that favors the tremor-driven-SSE model (See also Figure 19).

\subsection{RTR propagation distance, velocity, and possible implications}

Our extended study shows that, in some simulations, RTRs tend to slow down when they propagate far enough (e.g. Figures 10, 12 and 13). According to the proportionality between tremor migration speed and the background slow slip velocity (Equation 11), this slowdown reflects the spatial distribution of the slip velocity of the SSE pulse. This model feature is consistent with observations by Bletery et al. (2017) of a tendency of tremor migration to slow down further behind the SSE front. This suggests that RTR migration speed provides a constraint on the spatial distribution of slip velocity in an SSE, and the distance reached by RTRs constrains the width of an SSE pulse. 


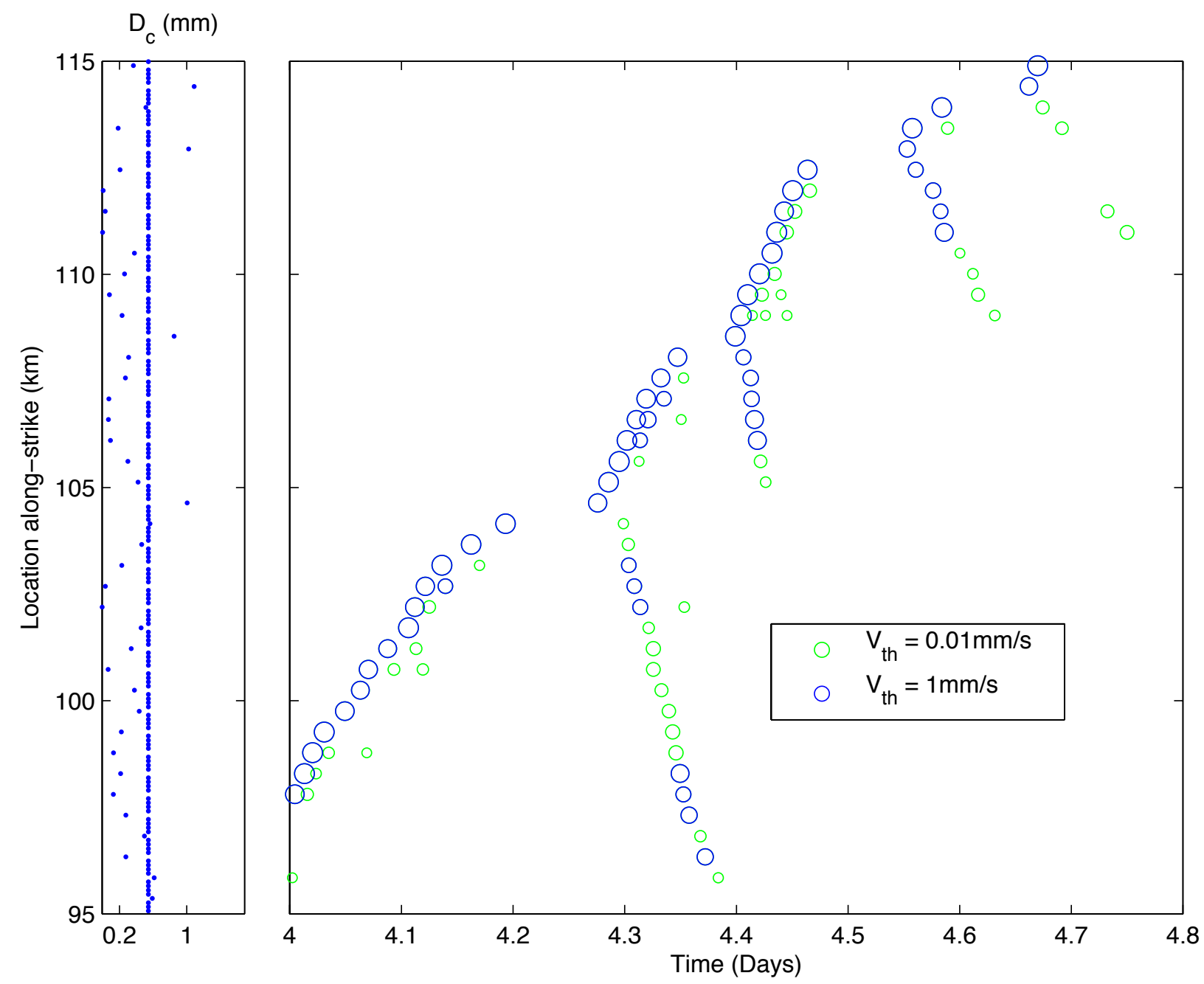

Figure 15: Nucleation of Rapid Tremor Reversals at "strong" asperities with large $D_{c}$ values.

\subsection{Tremor rate as a proxy for local slip rate}

As discussed in section 1, obtaining observations of local slip rate of SSE with high spatial and temporal resolution is very important, but challenging with conventional methods such as slip inversion based on GPS data. Our simulations show that the tremor rate is proportional to the local slip rate (measured within background matrix) (Figure 18). If this relation is valid in nature, we can infer slip rate with fine spatial-temporal resolution by monitoring the tremor activity rate. The relation may be calibrated for instance on the basis of coarse-scale slip inferred from geodetic observations. While in practice the analysis might not be straightforward, it offers a new perspective on existing data that may help us probe SSEs with unprecedented resolution.

\subsection{Comparison to previous models}

Several models have been previously proposed to explain subsets of observations of SSE and tremor. Here we discuss their relations to our model.

Various abstract models have been proposed to reproduce tremor signals. Ide et al. (2008)'s Brownian walk model reproduces the source time function of tremor swarms. Ide (2012) further developed the model to reproduce the large-scale tremor migration. Daub et al. (2011)'s fiber-bundle model reproduces the recurrence time and duration of tremor bursts. These models however do not reproduce hierarchical tremor migration patterns and the accompanying 


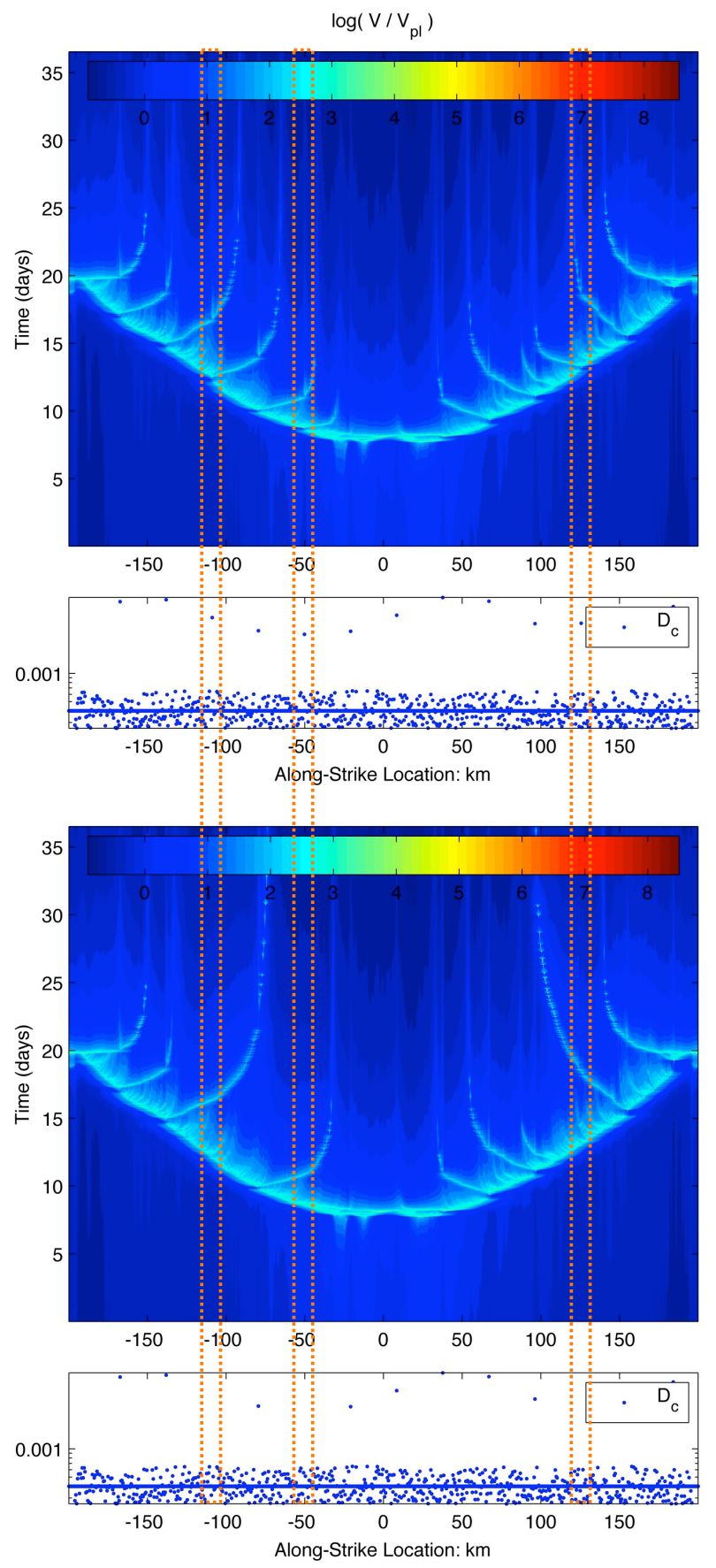

Figure 16: Test of the effect of strong asperities, analogous to Figure 10. Top: we set 10 very strong asperities with $D_{c}$ value about one order of magnitude larger than a normal asperity. All RTRs nucleate from these strong asperities. Bottom: we removed three of the strong asperities (indicated by orange blocks). The three RTRs disappeared. The other reversals that were either stopped or slowed down by these asperities are able to propagate faster/further beyond these removed strong asperities. Strong asperities not only nucleate tremor reversals but also acts as a barrier to slow down or even stop the RTR propagation.

\section{SSE.}

Attempts have also been made to build analogies between tremors and other phenomena. Gershenzon et al. (2011) 


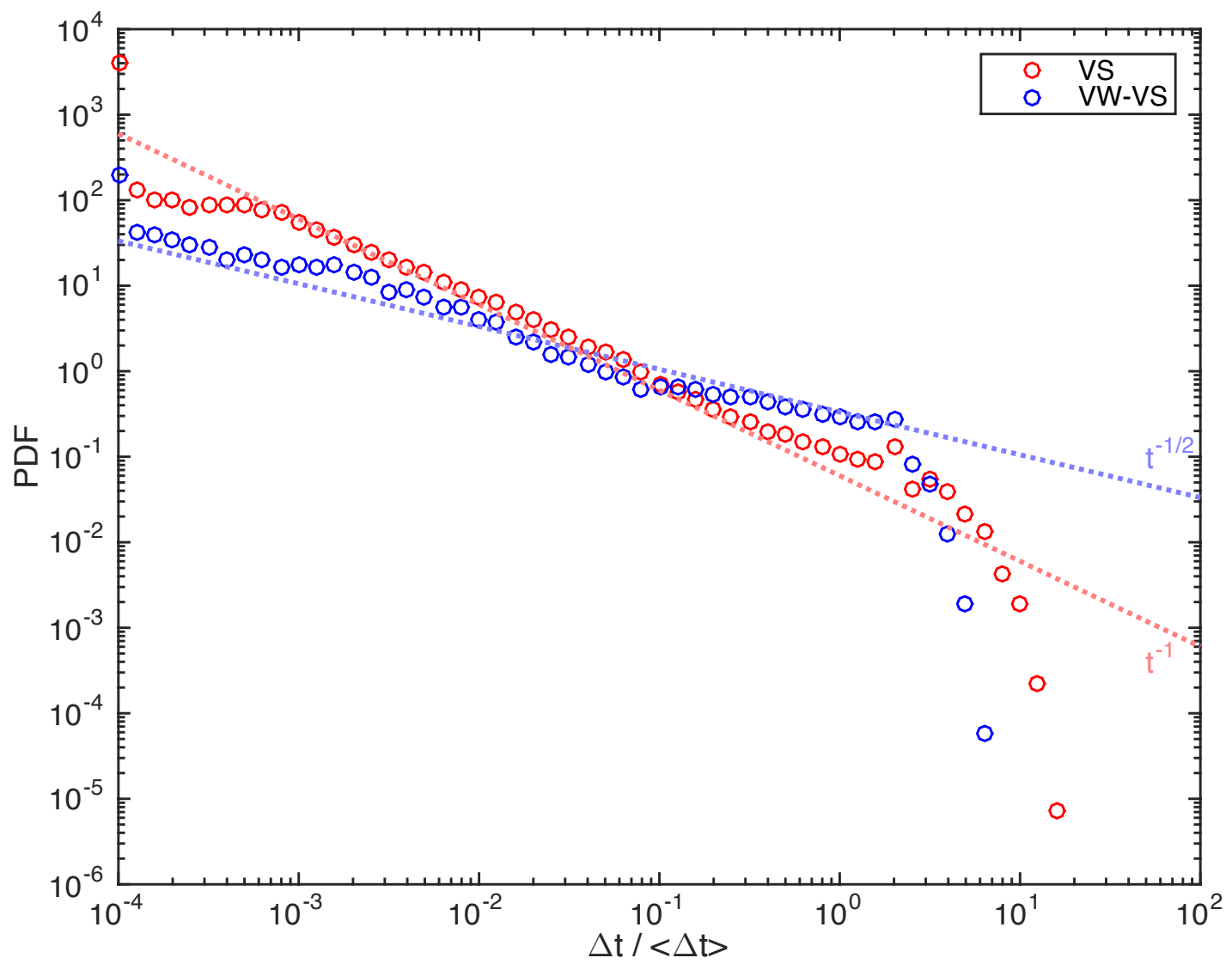

Figure 17: Probability density function of stacked tremor repeater recurrence interval distribution. Blue circles: SSEdriven-tremor model with friction with VW-VS transition in the matrix. Red circles: tremor-driven-SSE model with pure VS background. Both models have similar settings. Blue dashed line: $1 / \sqrt{t}$ decay. Red dashed line: $1 / t$ decay.

developed analogies between tremor migration patterns and the propagation of dislocations in crystals. Given the similarity between the pulse-like mode of slow slip propagation and a crystal dislocation, this analogy is not surprising. They then applied to SSE and tremor phenomena the Frenkel-Kontorova model of crystal dislocations, which is similar to a Burridge-Knopoff model of a chain of spring-blocks with a periodically slip-dependent friction. An ingredient of their model, worth considering in our future models, is a certain roughness effect which may represent the actual waviness of the fault caused by continued slip.

Discrete models without explicit asperities and creep have also been proposed. Ben-Zion (2012) treats tremor as the critical behavior of an inherently discrete model of a fault with zero net weakening. The model reproduces features of the incoherent background tremor activity in between SSEs, but not the organized migration patterns studied here. Colella et al. (2011, 2013)'s model reproduces the tremor migration patterns. Unlike our model, their model is inherently discrete and without quenched randomness. Thus the origin of RTR and along-dip tremor streaks in their model is not deterministic but stochastic, whereas our model reveals a clear mechanical origin of RTRs and along-dip tremor streaks. Also, the propagation velocity of RTRs and along-dip tremor streaks in their model are significantly slower than observations: both are at most 4 times the propagation velocity of the SSE, whereas observations are around 10 times and 100 times the SSE propagation velocity, respectively. The important difference is that in our model the asperities are embedded in a more stable fault matrix, so the tremor migration speeds are controlled by the propagation speed of transient creep between asperities, which in turn is directly proportional to the background slip velocity (section 2.2). In contrast, in their model the peak slip velocity is arbitrarily fixed.

Models without asperities but with creep have also been proposed. Hawthorne and Rubin (2013a,b) considered 


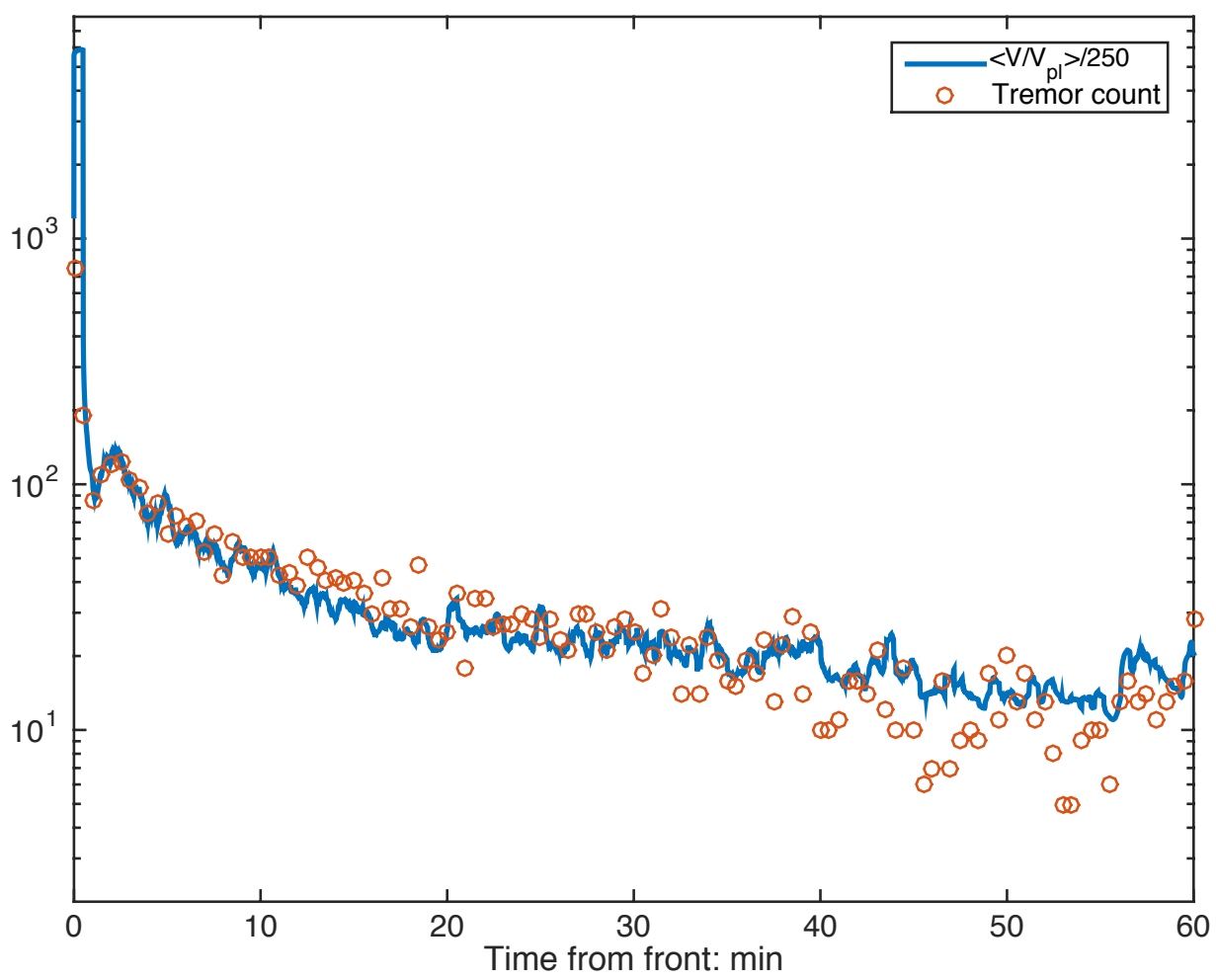

Figure 18: Tremor activity and local slip rate as a function of time during one tremor episode. Red circles: 30 second binned tremor activity as a function of time with respect to first local tremor activity. Blue solid curve: average local slip rate measured in matrix, aligned with local peak slip rate and low-pass filtered to $30 \mathrm{~s}$. The tremor rate and local slip rate are in strikingly good agreement.

friction with VW-VS transition at increasing velocity and simulated SSEs in quantitative agreement with observations. They also modeled reversal fronts triggered and modulated by tidal loading. The difference between their SSE model and ours has been discussed in section 2.3.1. The propagation speed of RTRs (and the $V_{R T R} / V_{S S E}$ ) in their model are at or below the lower values observed in Cascadia. Their model is not intended to simulate explicitly tremor activity. To develop further insight into tremor migration patterns, such models require additional assumptions about the relation between tremor rate and slow slip rate, which are evaluated in our model.

Homogeneous fault models with specially designed friction laws have also been considered. Rubin (2011) designed a rate-and-state friction law with two state variables to reproduce the different migration speeds of SSE along strike and along dip. However this model is not intended to reproduce tremor signals. Our models build upon earlier models of colledctive interaction of asperities: Ariyoshi et al. $(2009,2012)$ developed models of the whole subduction zone with a large megathrust asperity and tens of small asperities mimicking low frequency earthquake sources. In particular, Ariyoshi et al. (2012) introduced the important concept of swarms as a cascade of asperity failures. Also, Ando et al. $(2010,2012)$ presented an asperities-in-matrix model in which the rheology of the matrix is Newtonian viscosity. Their model produces Parabolic Tremor Migration (PTM), and RTRs and forward tremor migration are the collective appearance of superimposed PTMs. However, in their model RTRs are not faster than forward migration: Rapid Tremor Forward (RTF) occurs and propagates even faster and further than their RTRs. Because of the assumption of linear viscosity which, in contrast to a rate-and-state friction model, leads to tremor propagation speed uncorrelated to the background slip velocity (Ando et al. (2012); see also Ida (1974) and Savage (1971)), their model is not expected to produce the hierarchy of forward, reverse and along-dip tremor migration with distinct speeds and the slow-down of RTRs. The slower forward migration in their model is actually an apparent effect of overlapping PTMs, but observations in Southwest Japan do not support such PTM pattern. Moreover, In Cascadia, RTFs have not been 
reported: observations in Cascadia show a clear trend of tremors propagating along strike with a rather steady velocity (Figure 1).

\subsection{Model limitations and future work}

The quasi-dynamic approximation of elastodynamic effects adopted here is not a severe shortcoming, since the interaction of asperities in our model operates through slow creep fronts in the matrix, in which dynamic effects do not play an important role. Dynamic effects are more important inside asperities, but here we represented asperities as single cells without resolving the internal details of their rupture, which may not affect crucially the overall stress transfer to the creeping matrix that controls tremor migration patterns.

Whereas our conceptual model encompasses tremor migration along-strike and along-dip, the simulations presented here are 2D and are not intended to reproduce along-dip tremor migration. These 2D results do represent the essence of the conceptual model, as shown by its ability to reproduce forward and reverse along-strike tremor swarms with distinct migration speed. An animation of one of our 3D tremor and SSE simulations done with QDYN is shown in the supplemental materials. Further 3D results will be reported elsewhere that build up on the 2D results presented here, especially to set appropriate frictional properties and distributions of the asperities.

A further ingredient to consider in our model is non-planar fault geometry with anisotropic waviness, mimicking grooves aligned with the slip direction caused by wear and erosion of the megathrust interface. This can help address observations suggesting that the along-dip tremor migration is actually parallel to the ancient slip direction in some subduction zones (e.g. Ide 2012).

\section{Conclusions}

In this study, we developed mechanical models that reproduce imbricated tremor migration patterns along-strike accompanying slow slip events. The models represent a collection of frictionally unstable asperities embedded in a frictionally stable fault zone matrix, to investigate the role of fault heterogeneity and multi-asperity interactions mediated by creep. We focused on two models representing end-member behaviors: the SSE-driven-tremor model and the tremor-driven-SSE model. Both models reproduce qualitatively and quantitatively a broad range of observed characteristics of slow slip and tremor. In particular, they reproduce the distinct migration speed of forward tremor propagation and rapid tremor reversals (RTRs) and provide a mechanical relation between tremor migration speed and the slip velocity of the underlying slow slip.

Additional main findings of this work are as follows. (1) A model of SSE driven by tremor is viable, and seems favored by observations over the conventional model of tremor driven by SSE. While both models reproduce observed characteristics of SSE and tremor quantitatively, the tremor-driven-SSE model is based on a more conventional friction law and reproduces key observations better and without fine-tuning, in particular the range of RTR to SSE migration speeds and the decay of LFE inter-event times. (2) Tremor activity sheds light on fault rheology. The hierarchical patterns of tremor migration, with distinct propagation speeds in different directions, rule out a linear viscous rheology of the fault matrix and favor non-linear rheologies such as rate-and-state friction. The initiation points of RTRs also shed light on fault rheology at a fine scale: RTRs tend to nucleate at asperities that are stronger. Comparing RTR locations over multiple tremor episodes can probe temporal changes of local asperity strength which may be related, for instance, to changes of pore pressure. (3) A heterogeneous fault with a mixture of velocity-strengthening and velocity-weakening materials can generate SSEs robustly. This mechanism of SSE generation works without fine tuning, even under classical rate-and-state friction and with the slip law for state evolution, over a range of model parameter values much wider than in a homogeneous fault. It is enabled by collective interactions on heterogeneous faults with a broad range of stochastic asperity properties. (4) Tremor activity may be used to monitor SSE properties at a fine scale. Despite strong interactions between asperities, our models yield a simple relation between tremor rate and the underlying slip rate. This provides quantitative support for an approach to constrain SSE slip rate at high spatial and temporal resolution through observations of tremor rates. 


\section{References}

Ampuero, J. P. and A. M. Rubin (2008), "Earthquake nucleation on rate and state faults-Aging and slip laws". Journal of Geophysical Research: Solid Earth, 113 (B1). B01302.

Ampuero, J. P., O. Lengliné, Y. Luo, V. Durand, and J. A. Ruiz (2014), "Segmentation and Large-Scale Nucleation of the 2014 Pisagua Earthquake Sequence". AGU Fall Meeting Abstracts.

Ando, R., R. Nakata, and T. Hori (2010), "A slip pulse model with fault heterogeneity for low-frequency earthquakes and tremor along plate interfaces". Geophysical Research Letters, 37 (10). L10310.

Ando, R., N. Takeda, and T. Yamashita (2012), "Propagation dynamics of seismic and aseismic slip governed by fault heterogeneity and Newtonian rheology". Journal of Geophysical Research: Solid Earth, 117 (B11). B11308.

Ariyoshi, K., T. Hori, J. P. Ampuero, Y. Kaneda, T. Matsuzawa, R. Hino, and A. Hasegawa (2009), "Influence of interaction between small asperities on various types of slow earthquakes in a 3-D simulation for a subduction plate boundary". Gondwana Research, 16 (3), pp. 534-544.

Ariyoshi, K., T. Matsuzawa, J. P. Ampuero, R. Nakata, T. Hori, Y. Kaneda, R. Hino, and A. Hasegawa (2012), "Migration process of very low-frequency events based on a chain-reaction model and its application to the detection of preseismic slip for megathrust earthquakes". Earth, Planets and Space, 64 (8), pp. 693-702.

Bebout, G. E. and M. D. Barton (2002), "Tectonic and metasomatic mixing in a high-T, subduction-zone mélange—insights into the geochemical evolution of the slab-mantle interface". Chemical Geology, 187 (1), pp. 79-106.

Ben-Zion, Y. (2012), "Episodic tremor and slip on a frictional interface with critical zero weakening in elastic solid". Geophysical Journal International, 189 (2), pp. 1159-1168.

Beroza, G. C. and S. Ide (2011), "Slow earthquakes and nonvolcanic tremor". Annual Review of Earth and Planetary Sciences, 39, pp. 271-296.

Bhattacharya, P. and A. M. Rubin (2014), "Frictional response to velocity steps and 1-D fault nucleation under a state evolution law with stressing-rate dependence". Journal of Geophysical Research: Solid Earth, 119 (3), pp. 22722304.

Bletery, Q., A. M. Thomas, J. C. Hawthorne, R. M. Skarbek, A. W. Rempel, and R. D. Krogstad (2017), "Characteristics of secondary slip fronts associated with slow earthquakes in Cascadia". Earth and Planetary Science Letters, 463, pp. 212-220.

Chen, K. H., R. M. Nadeau, and R. J. Rau (2007), "Towards a universal rule on the recurrence interval scaling of repeating earthquakes?" Geophysical Research Letters, 34 (16). L16308.

Colella, H. V., J. H. Dieterich, and K. Richards-Dinger (2011), "Multi-event simulations of slow slip events for a Cascadia-like subduction zone". Geophysical Research Letters, 38 (16). L16312.

- (2013), "Spatial and temporal patterns of simulated slow slip events on the Cascadia megathrust". Geophysical Research Letters, 40 (19), pp. 5101-5107.

Daub, E. G., D. R. Shelly, R. A. Guyer, and P. A. Johnson (2011), "Brittle and ductile friction and the physics of tectonic tremor". Geophysical Research Letters, 38 (10). L10301.

Dieterich, J. H. (1979), "Modeling of rock friction: 1. Experimental results and constitutive equations". Journal of Geophysical Research: Solid Earth, 84 (B5), pp. 2161-2168.

Dublanchet, P., P. Bernard, and P. Favreau (2013), "Interactions and triggering in a 3-D rate-and-state asperity model". Journal of Geophysical Research: Solid Earth, 118 (5), pp. 2225-2245.

Fagereng, Å. (2011), "Fractal vein distributions within a fault-fracture mesh in an exhumed accretionary mélange, Chrystalls Beach Complex, New Zealand”. Journal of Structural Geology, 33 (5), pp. 918-927.

Fagereng, Å. and A. F. Cooper (2010), "The metamorphic history of rocks buried, accreted and exhumed in an accretionary prism: an example from the Otago Schist, New Zealand". Journal of Metamorphic Geology, 28 (9), pp. $935-954$.

Fagereng, Å. and R. H. Sibson (2010), “Melange rheology and seismic style”. Geology, 38 (8), pp. 751-754.

Field, E. H. et al. (2017), "A Synoptic View of the Third Uniform California Earthquake Rupture Forecast (UCERF3)". Seismological Research Letters, pp. 1259-1267.

Gershenzon, N. I., G. Bambakidis, E. Hauser, A. Ghosh, and K. C. Creager (2011), "Episodic tremors and slip in Cascadia in the framework of the Frenkel-Kontorova model". Geophysical Research Letters, 38 (1). L01309.

Ghosh, A., J. E. Vidale, J. R. Sweet, K. C. Creager, A. G. Wech, H. Houston, and E. E. Brodsky (2010), "Rapid, continuous streaking of tremor in Cascadia". Geochemistry, Geophysics, Geosystems, 11 (12). Q12010.

Gomberg, J. et al. (2010), "Slow-slip phenomena in Cascadia from 2007 and beyond: A review". Geological Society of America Bulletin, 122 (7-8), pp. 963-978. 
Hawthorne, J. C. and A. M. Rubin (2010), "Tidal modulation of slow slip in Cascadia". Journal of Geophysical Research: Solid Earth, 115 (B9). B09406.

- (2013a), "Laterally propagating slow slip events in a rate and state friction model with a velocity-weakening to velocity-strengthening transition”. Journal of Geophysical Research: Solid Earth, 118 (7), pp. 3785-3808.

- (2013b), "Tidal modulation and back-propagating fronts in slow slip events simulated with a velocity-weakening to velocity-strengthening friction law". Journal of Geophysical Research: Solid Earth, 118 (3), pp. 1216-1239.

Horowitz, F. G. and A. Ruina (1989), "Slip patterns in a spatially homogeneous fault model”. Journal of Geophysical Research: Solid Earth, 94 (B8), pp. 10279-10298.

Houston, H., B. G. Delbridge, A. G. Wech, and K. C. Creager (2011), "Rapid tremor reversals in Cascadia generated by a weakened plate interface". Nature Geoscience, 4 (6), p. 404.

Ida, Y. (1974), "Slow-moving deformation pulses along tectonic faults". Physics of the Earth and Planetary Interiors, 9 (4), pp. 328-337.

Ide, S. (2012), "Variety and spatial heterogeneity of tectonic tremor worldwide". Journal of Geophysical Research: Solid Earth, 117 (B3). B03302.

Ide, S., G. C. Beroza, D. R. Shelly, and T. Uchide (2007a), “A scaling law for slow earthquakes”. Nature, 447 (7140), p. 76.

Ide, S., D. R. Shelly, and G. C. Beroza (2007b), "Mechanism of deep low frequency earthquakes: Further evidence that deep non-volcanic tremor is generated by shear slip on the plate interface". Geophysical Research Letters, 34 (3). L03308.

Ide, S., K. Imanishi, Y. Yoshida, G. C. Beroza, and D. R. Shelly (2008), "Bridging the gap between seismically and geodetically detected slow earthquakes". Geophysical Research Letters, 35 (10). L10305.

Ito, Y., K. Obara, T. Matsuzawa, and T. Maeda (2009), "Very low frequency earthquakes related to small asperities on the plate boundary interface at the locked to aseismic transition". Journal of Geophysical Research: Solid Earth, 114 (B11). B00A13.

Kao, H., S. Shan, H. Dragert, and G. Rogers (2009), "Northern Cascadia episodic tremor and slip: A decade of tremor observations from 1997 to 2007”. Journal of Geophysical Research: Solid Earth, 114 (B11). B00A12.

Kao, H., K. Wang, H. Dragert, J. Kao, and G. Rogers (2010), "Estimating seismic moment magnitude (Mw) of tremor bursts in northern Cascadia: Implications for the "seismic efficiency" of episodic tremor and slip". Geophysical Research Letters, 37 (19). L19306.

Kato, A., K. Obara, T. Igarashi, H. Tsuruoka, S. Nakagawa, and N. Hirata (2012), "Propagation of slow slip leading up to the $2011 \mathrm{Mw} 9.0$ Tohoku-Oki earthquake". Science, 335 (6069), pp. 705-708.

Kato, N. (2007), "Expansion of aftershock areas caused by propagating post-seismic sliding”. Geophysical Journal International, 168 (2), pp. 797-808.

Lay, T., H. Kanamori, C. J. Ammon, K. D. Koper, A. R. Hutko, L. Ye, H. Yue, and T. M. Rushing (2012), "Depthvarying rupture properties of subduction zone megathrust faults". Journal of Geophysical Research: Solid Earth, 117 (B4). B04311.

Lengliné, Olivier, WB Frank, D Marsan, and J-P Ampuero (2017), "Imbricated slip rate processes during slow slip transients imaged by low-frequency earthquakes". Earth and Planetary Science Letters, 476, pp. 122-131.

Liu, Y. and J. R. Rice (2005), “Aseismic slip transients emerge spontaneously in three-dimensional rate and state modeling of subduction earthquake sequences”. Journal of Geophysical Research: Solid Earth, 110 (B8). B08307.

Lui, S. K. Y. and N. Lapusta (2016), "Repeating microearthquake sequences interact predominantly through postseismic slip". Nature communications, 7, p. 13020.

Luo, Y. and J. P. Ampuero (2017), "Stability of faults with heterogeneous friction properties and effective normal stress".

Luo, Y., J. P. Ampuero, P. Galvez, M. Ende, and B. Idini (2017), QDYN: a Quasi-DYNamic earthquake simulator (v1.1). DOI: 10.5281/zenodo.322459. URL: https://doi .org/10.5281/zenodo. 322459.

Marone, C. (1998), "The effect of loading rate on static friction and the rate of fault healing during the earthquake cycle". Nature, 391 (6662), p. 69.

Meneghini, F., G. Di Toro, C. D. Rowe, J. C. Moore, A. Tsutsumi, and A. Yamaguchi (2010), "Record of megaearthquakes in subduction thrusts: the black fault rocks of Pasagshak Point (Kodiak Island, Alaska)". Geological Society of America Bulletin, 122 (7-8), pp. 1280-1297.

Meng, L., A. Inbal, and J. P. Ampuero (2011), “A window into the complexity of the dynamic rupture of the $2011 \mathrm{Mw}$ 9 Tohoku-Oki earthquake”. Geophysical Research Letters, 38 (7). L00G07. 
Peng, Y., A. M. Rubin, M. G. Bostock, and J. G. Armbruster (2015), "High-resolution imaging of rapid tremor migrations beneath southern Vancouver Island using cross-station cross correlations". Journal of Geophysical Research: Solid Earth, 120 (6), pp. 4317-4332.

Peng, Z. and J. Gomberg (2010), "An integrated perspective of the continuum between earthquakes and slow-slip phenomena". Nature Geoscience, 3 (9), pp. 599-607.

Perfettini, H. and J. P. Ampuero (2008), "Dynamics of a velocity strengthening fault region: Implications for slow earthquakes and postseismic slip". Journal of Geophysical Research: Solid Earth, 113 (B9). B09411.

Rice, J. R. (1993), "Spatio-temporal complexity of slip on a fault". Journal of Geophysical Research: Solid Earth, 98 (B6), pp. 9885-9907.

Rogers, G. and H Dragert (2003), "Episodic tremor and slip on the Cascadia subduction zone: The chatter of silent slip". Science, 300 (5627), pp. 1942-1943.

Rolandone, F., R. Bürgmann, and R. M. Nadeau (2004), "The evolution of the seismic-aseismic transition during the earthquake cycle: Constraints from the time-dependent depth distribution of aftershocks". Geophysical Research Letters, 31 (23). L23610.

Rouet-Leduc, B., C. Hulbert, N. Lubbers, K. Barros, C. Humphreys, and P. A. Johnson (2017), "Machine Learning Predicts Laboratory Earthquakes". arXiv preprint arXiv:1702.05774. LAUR 1808407.

Rubin, A. M. (2008), "Episodic slow slip events and rate-and-state friction". Journal of Geophysical Research: Solid Earth, 113 (B11). B11414.

- (2011), "Designer friction laws for bimodal slow slip propagation speeds". Geochemistry, Geophysics, Geosystems, 12 (4). Q04007.

Rubin, A. M. and J. P. Ampuero (2005), "Earthquake nucleation on (aging) rate and state faults". Journal of Geophysical Research: Solid Earth, 110 (B11). B11312.

Ruina, A. (1983), "Slip instability and state variable friction laws". Journal of Geophysical Research: Solid Earth, 88 (B12), pp. 10359-10370.

Ruiz, S., M. Metois, A. Fuenzalida, J. Ruiz, F. Leyton, R. Grandin, C. Vigny, R. Madariaga, and J. Campos (2014), "Intense foreshocks and a slow slip event preceded the 2014 Iquique Mw 8.1 earthquake". Science, 345 (6201), pp. 1165-1169.

Savage, L. J. (1971), "Elicitation of personal probabilities and expectations". Journal of the American Statistical Association, 66 (336), pp. 783-801.

Segall, P., A. M. Rubin, A. M. Bradley, and J. R. Rice (2010), "Dilatant strengthening as a mechanism for slow slip events". Journal of Geophysical Research: Solid Earth, 115 (B12). B12305.

Shelly, D. R., G. C. Beroza, S. Ide, and S. Nakamula (2006), "Low-frequency earthquakes in Shikoku, Japan, and their relationship to episodic tremor and slip". Nature, 442 (7099), p. 188.

Shelly, D. R., G. C. Beroza, and S. Ide (2007), "Complex evolution of transient slip derived from precise tremor locations in western Shikoku, Japan”. Geochemistry, Geophysics, Geosystems, 8 (10). Q10014.

Shibazaki, B. and Y. Iio (2003), "On the physical mechanism of silent slip events along the deeper part of the seismogenic zone". Geophysical Research Letters, 30 (9). 1489.

Skarbek, R. M., A. W. Rempel, and D. A. Schmidt (2012), "Geologic heterogeneity can produce aseismic slip transients". Geophysical Research Letters, 39 (21). L21306.

Wech, A. G., K. C. Creager, H. Houston, and J. E. Vidale (2010), "An earthquake-like magnitude-frequency distribution of slow slip in northern Cascadia". Geophysical Research Letters, 37 (22). L22310.

Yabe, S. and S. Ide (2017), "Slip-behavior transitions of a heterogeneous linear fault". Journal of Geophysical Research: Solid Earth, 122 (1), pp. 387-410.

An animation preview of 3D tremor migrations:

https : //github.com/ydluo/qdyn/wiki/Simulation_Cascadia_Tremor 


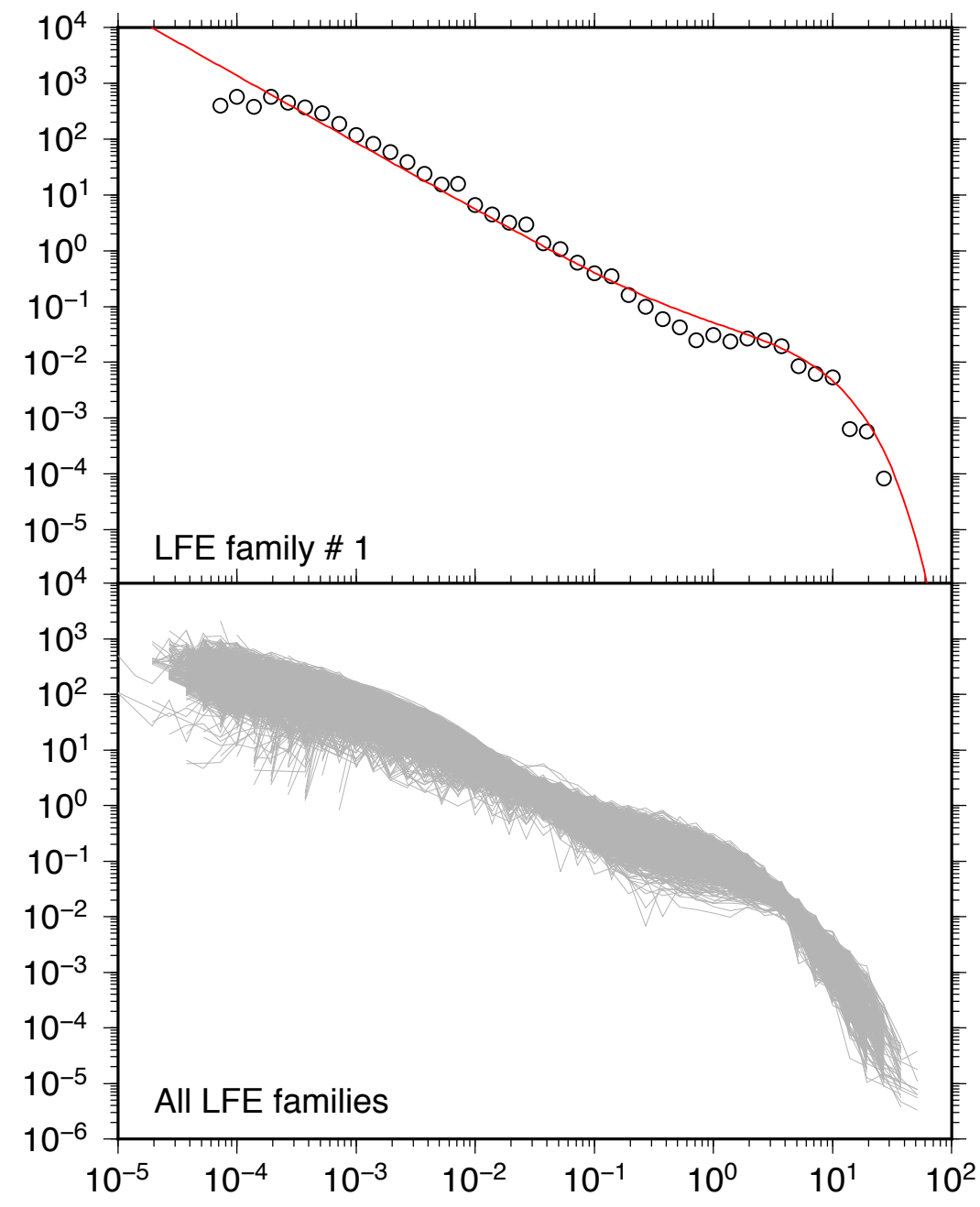

Figure 19: (From written communication with O. Lengliné 2015, see also Lengliné et al. (2017) supplemental materials). Probability density function of recurrence intervals of LFE families from Mexico, analogous to Figure 17. Top: repeater recurrence interval distribution from a selected LFE family in Mexico subduction (black circles) and fit (red curve), the fit shows a transition from a power-law decay with an exponent $\mathrm{q}=1.0(1 / t)$ at short time scale to an exponential decay. Bottom: superposed pdfs computed for all families (gray curves). The same shape of the pdf is recovered for all families. 